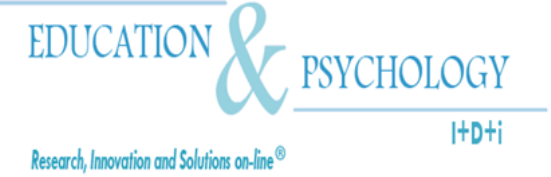

\title{
Origen y desarrollo del pensamiento numérico: una perspectiva multidisciplinar
}

\section{José Domingo Villarroel Villamor}

Escuela Universitaria de Magisterio de Vitoria, Universidad del País Vasco, Vitoria

\section{España}

José Domingo Villarroel Villamor. Escuela Universitaria de Magisterio de Vitoria. Universidad del País Vasco. Juan Ibáñez de Sto. Domingo, 1. 01006 Vitoria. España. E-mail: txomin.villarroel@ehu.es

(C) Education \& Psychology I+D+i and Editorial EOS (Spain) 


\section{Resumen}

Se presenta una revisión de la investigación que sobre la cuestión del origen y desarrollo del pensamiento numérico se ha realizado desde distintos paradigmas. Se aborda esta cuestión desde la perspectiva de la psicología experimental, de los estudios de cognición comparada, de las modernas técnicas de neuroimagen y, finalmente, considerando las aportaciones de la psicología del desarrollo.

A lo largo del ensayo se pretende exponer que la desafiante cuestión de saber cómo los seres humanos desarrollamos la habilidad de pensar con y sobre números es también, una materia compleja que indefectiblemente exige un abordaje multidisciplinar.

La revisión termina reflexionando sobre las aportaciones que los diversos puntos sobre el pensamiento numérico ofrecen para el diseño de prácticas educativas, las cuales, necesariamente, también deben ser abordadas desde un perspectiva ecléctica.

Palabras Clave: Pensamiento numérico, Neurociencias, Psicología cognitiva, Psicología experimental, Estudios comparados.

Recibido: 28/07/08 Aceptación inicial: 01/09/08 Aceptación final: 26/12/08 


\begin{abstract}
A review of research on the matter of the origin and development of numerical thinking is presented from different paradigms.

This topic is dealt from the perspective of experimental psychology, comparative studies of cognition, of modern neuroimaging techniques and, finally, considering the contributions of developmental psychology.

The essay aims to expose the challenging subject of how humans develop the ability to think about and with numbers but this matter is also a complex one that inevitably requires a multidisciplinary approach.

The review concludes by reflecting on the contributions that different points of view offer about numerical thinking for designing educational practices, which necessarily must also be addressed from an eclectic perspective.
\end{abstract}

Keywords: Numerical thinking, Neuro-sciences, Cognitive Psychology, Experimental Psychology,

Received: 07/28/08 Initial Acceptance: 09/01/08 Final Acceptance: 12/26/08 


\section{Introducción}

Ciertamente, resulta innecesario destacar la capacidad de utilizar números como una de las habilidades cognitivas indispensables para la adecuada adaptación de la ciudadanía a un entorno cultural caracterizado por la abundancia de información cuantitativa. Precisamente, esta importancia que tienen las habilidades numéricas en los procesos de adaptación y desarrollo de las personas e igualmente, la universalidad característica de la cognición numérica a lo largo de culturas y civilizaciones han propiciado que sea una cuestión de innegable interés científico el estudio de cómo los seres humanos desarrollamos la capacidad de seleccionar los aspectos cuantitativos de los estímulos y cómo, en torno a ellos, generamos entidades abstractas sobre los que desplegamos una vasta actividad intelectual y comunicativa.

En este ámbito de conocimiento surgen cuestiones de gran calado científico relativas, por ejemplo, a la comprensión del proceso que permite a los niños aprehender el significado de los números, al dilema sobre si existe o no, un fundamento innato en dicho proceso y al papel que las destrezas lingüísticas juegan en la aprehensión de estos significados (Díaz, 2006; Feigenson, Dehaene y Spelke, 2004).

Igualmente desafiante resulta cuestionarse por el fundamento neurológico del entendimiento numérico, si realmente existe algún grupo neuronal preferente que dirige este pensamiento y qué parte de estas bases neuronales y sus correspondientes habilidades son compartidas por otros primates no-humanos (Alonso y Fuentes; 2001; Brannon, 2006; Dehaene, Molko, Cohen y Wilson 2004).

Por otro lado, y al margen de estos retos intelectuales, no se puede dejar pasar por alto la enorme transcendencia que los avances en la comprensión del origen y desarrollo del pensamiento numérico deberían tener en el diseño de intervenciones educativas eficaces, especialmente a edades tempranas (Barth, La Mont, Lipton y Spelke, 2005).

Considerando la amplitud de las cuestiones expuestas no resulta extraño que el estudio del origen y desarrollo de la habilidad para representar números haya sido una materia abordada desde distintos campos del conocimiento: las neurociencias (Gracia-Bafalluy y Noël, 2008; Klessinger; Szczerbinskia, Varley, 2007), la psicología (Berg, 2008; Condry y Spelke, 2008), la lingüística (Campbell y Lynette, 2004; Campbell y Metcalfea, 2008; Corvera, Doet- 
jesb y Zwartsa, 2007), la antropología (De Cruz, 2006; Sheridan, 2005) y la propia didáctica de la matemática (Ebersbach, Luwel, Frick, Onghena y Verschaffel, 2008; Gilmore y Spelke, en prensa).

A lo largo de este artículo se presenta una revisión de las aportaciones más actuales sobre las cuestiones anteriormente expuestas, relativas a cómo los seres humanos desarrollamos la capacidad de pensar y comunicar sobre cualidades cuantitativas de la realidad. Se analizarán, especialmente, tres aspectos de la investigación en este campo: los estudios relativos a las regularidades conductuales características de los seres humanos cuando pensamos con números; las aportaciones que el estudio en profundidad del córtex cerebral está generando en torno a qué zonas neuronales se relacionan con el pensamiento numérico y, finalmente, cómo este pensamiento evoluciona a lo largo del desarrollo.

\section{El pensamiento numérico desde la psicología experimental}

La idea de que es posible identificar componentes psíquicos a través de la medición de los tiempos de reacción en pruebas de complejidad variable es un supuesto teórico que sustenta un gran número de investigaciones en el campo de la psicología experimental desde que en 1860 el físico alemán Gustav Theodor Fechner publico su obra "Elementos de psicofísica".

Dentro del ámbito de la cognición numérica también se ha desarrollado esta línea de investigación, especialmente a partir de la década de los 60 del pasado siglo, momento en el que comenzaron a surgir diversas iniciativas que trataban de establecer las características de la representación de los números que el cerebro elabora.

Estas investigaciones se han centrado, fundamentalmente, en el análisis de las respuestas conductuales de los individuos. De esta manera y estudiando los tiempos de reacción en tareas vinculadas a estímulos numéricos, se han puesto de manifiesto fenómenos relacionados con el pensamiento numérico que permiten esbozar cómo pueden ser las representaciones internas que los individuos crean entorno a los números. Estos fenómenos, que se presentan a continuación son: el efecto distancia, el efecto tamaño y la ordenación espacial numérica o efecto SNARC. 


\section{El efecto distancia}

Un fenómeno característico, aunque no exclusivo, del procesamiento numérico se refiere al hecho de que al comparar dos números el tiempo necesario para discriminar el mayor del menor, disminuye al incrementarse la distancia entre los números. De esta manera, estadísticamente se invierte un lapso de tiempo mayor en diferenciar el mayor de 2 y 4 que 2 y 9. A este efecto se le conoce como el efecto distancia (Moyer y Landauer, 1967; Rubinsten, Henik, Berger y Shahar-Shalev, 2002).

En el contexto de la cognición numérica, este fenómeno se ha interpretado como una consecuencia de la interferencia en la activación de las representaciones de los números a medida que disminuye la diferencia entre su valores absolutos y, asimismo, se ha relacionado con la idea de que la imagen que el cerebro elabora en torno a las cantidades se ajusta más a una representación de magnitudes continúas que a la de elementos discretos (Cappelletti y al., 2007; Göbel y Rushworth, 2004) o meras representaciones simbólicas.

El efecto distancia ha sido corroborado tanto en el caso de comparaciones de cantidades expresadas simbólicamente, a través de dígitos, como cantidades expresadas no simbólicamente, es decir, mediante conjuntos de objetos (Cappelletti et al., 2007) y, en esta última línea de investigación, ha sido observado no sólo en adultos sino también en infantes (Rubinsten, Henik, Berger y Shahar-Shalev, 2002) e incluso en animales (Dehaene, DehaeneLambertz y Cohen, 1998; Emmerton, Lohmann y Niemann, 1997). También, se ha registrado en el caso del procesamiento de magnitudes continuas como, por ejemplo, el peso (Pinel, Piazza, Le Bihan y Dehaene, 2004).

Este fenómeno ha sido considerado como una evidencia de que un único mecanismo subyace en el procesamiento cognitivo de magnitudes y cantidades expresadas simbólica y no simbólicamente (Dehaene et al., 2003; Gelman y Gallistel, 2004) de forma que "los individuos convierten los estímulos numéricos (signos escritos u oídos) en magnitudes continuas. La comparación entre estas magnitudes se lleva a cabo de la misma manera que las comparaciones entre estímulos físicos, tales como la longitud de líneas. Se entiende que la causa del efecto distancia es el solapamiento entre las representaciones numéricas" (Rubinsten et al., 2002, p.1). 


\section{El efecto tamaño}

Otro efecto que aporta información en torno a cómo el cerebro representa los números es el denominado efecto tamaño (Restle, 1970). Lo que se registra en el estudio de este fenómeno es que el tiempo empleado en la discriminación del mayor de dos números aumenta según se incrementa el valor absoluto de los números considerados y que esta capacidad de discriminación obedece, como el caso de otro tipo de estímulos, a la ley de Weber (2007). En consecuencia, discernir qué número es mayor en el par 18 y 20 requiere más tiempo que en el caso de que los números propuestos sean 1 y 3 , y esto a pesar de que la diferencia absoluta entre ambos sea la misma.

La interpretación más aceptada de este fenómeno es que la discriminación entre números empeora a medida que crece la magnitud que representan porque disminuye la distancia subjetiva entre los mismos (Longo y Lourenco, 2007).

Ambos dos efectos mencionados (distancia y tamaño) han sido referidos como independientes del lenguaje y del tipo de notación empleada. Incluso se constata, a partir de estudios realizados en poblaciones indígenas Brasileñas sin contacto con nuestro sistema numérico y con lenguajes parvos en cuanto al empleo de palabras-número, que la precisión para referirse a cantidades obedece a la ley de Weber resultando que la capacidad de discernimiento de cantidades depende del radio entre ambas y no de los valores absolutos (Brannon, 2006).

\section{La ordenación espacial numérica o efecto SNARC}

EL efecto SNARC (Spatial-Numerical Association of Response Codes) se constató a través de experimentos en los que el participante debía juzgar la naturaleza par o impar de dígitos y en los que se medía el tiempo de reacción para ejecutar la respuesta a partir del movimiento de la mano, por ejemplo, pulsando una tecla (Dehaene Bossini y Giraux, 1993).

Estas investigaciones muestran que existen diferencias en los tiempos de reacción de cada mano, de forma que se generan respuestas más rápidas y con una menor frecuencia de errores para números pequeños con la mano izquierda, con relación a los tiempos empleados con la mano derecha. Sin embargo, con números grandes, el efecto es inverso (Gevers y Lammertyn, 2005). 
El efecto SNARC que ha sido descrito tanto en personas diestras como zurdas; se ha constatado, incluso, con los brazos cruzados (Alonso y Fuentes, 2001) y únicamente muestra variaciones en poblaciones árabes monolingües alfabetizadas en las que la orientación de la línea discurre de derecha a izquierda (Zebian, 2005).

A través de estos experimentos se demuestra que la magnitud inherente a un dígito, que, a priori, es una característica irrelevante con relación a su paridad, realmente, sí interfieren en la velocidad de juicio sobre la misma.

Los resultados derivados del estudio del efecto SNARC han sido interpretados como una prueba de que en la búsqueda de una respuesta a juicios relativos a la naturaleza de los números, el individuo accede a una representación espacial interna de los mismos que, en último término, condiciona la velocidad de ejecución de las respuestas. Esta asociación número-espacial es concebida como un modelo o representación semejante a una línea o continuum que discurre orientado de izquierda a derecha y en el que cada número corresponde a un punto de la misma (Gevers et al., 2006; Mapelli, Rusconi y Umilta, 2003; Saal, Izquierdo y Codnia 2005).

Este fenómeno ha resultado no ser específico de la cognición numérica ya que ha sido descrito en el estudio de otro tipo de magnitudes de naturaleza continua como el tiempo o al sonido (Cohen, Lammertyn y Izard, 2008; González-Hernández, 2007; Rusconi, Kwana, Giordano, Umilta y Butterworth, 2006).

En conjunto, los efectos anteriormente expuestos sugieren un modelo cognitivo que evidenciaría, por un lado, la naturaleza fundamentalmente espacial de las representación semántica de los números y, por otro, el hecho de que los mecanismos cognitivos subyacentes a estas representaciones (o, tal vez, las propias representaciones) podrían ser elementos comunes en el procesamiento de magnitudes discretas y continuas (Cohen et al., 2008).

Como veremos posteriormente, esta relación entre orientación espacial y pensamiento numérico quedará evidenciada también, desde otras perspectivas metodológicas, lo que sin duda, resultaría ser un hallazgo de importantes repercusiones educativas. 


\section{El pensamiento numérico desde las neurociencias}

A lo largo del siglo XX, el estudio de determinadas lesiones cerebrales que de forma específica afectan a la capacidad de manejar números, los datos relativos a las habilidades numéricas pre-lingüísticas de bebes y el análisis de las destrezas cognitivas que demuestran ciertas especies animales, permitieron sugerir que debiera existir algún sustrato biológico determinable que actúe como precursor de las capacidades numéricas.

Estos planteamientos novedosos han promovido es estudio de las estructuras anatómicas y funcionales que sustentan la base competencial de las habilidades numéricas y han promocionado la investigación desde tres puntos de vista metodológicos, los vinculados al análisis de las patologías y daño cerebral, los estudios de cognición numérica comparada y las modernas técnicas de neuroimagen.

\section{El sindrome de Gerstmann}

Entre 1924 y 1930 el neurólogo austriaco Josef Gerstmann (1887- †1969) publicó tres artículos describiendo lo que consideraba un nuevo síndrome caracterizado por agnosia digital, agrafia, acalculia y desorientación derecha-izquierda (Gerstmann, 1940).

La agnosia digital se refiere a la imposibilidad de reconocer los propios dedos de forma que cuando un paciente sufre este síndrome comete errores evidentes para nombrar sus dedos o los de otra persona o para identificar los dedos a partir del nombre. Este síntoma, sin embargo, aparece exclusivamente vinculado a los dedos, no manifestándose dificultades en el reconocimiento de otras partes del cuerpo (Lebrun, 2005).

La acalculia se refiere a un trastorno específico para el cálculo y manejo de números y no se corresponde con un mero desorden de aprendizaje. Esta afección fue descrita por Henschen en 1919 y fue este mismo neurólogo quien subrayó la necesidad de considerar la existencia de un sistema cerebral específicamente encargado del cálculo, independiente, del sistema del lenguaje (Kahn, 1991).

Finalmente, y con relación al síndrome de Gerstmann, éste ocasiona, también, agrafia, un déficit relacionado con dificultades o imposibilidad para manejar símbolos escritos y comunicar ideas a través de ellos (Balasubramanian, 2006). 
El origen del síndrome de Gerstmann se sitúa en lesiones en la zona parietal inferior izquierda (Lebrun, 2005) y se ha descrito que pudiera reflejar la existencia de redes neuronales con cometidos altamente especializados en el manejo de números, dedos, orientación espacial y escritura (Alonso y Fuentes, 2001).

En esta línea se ha constatado que la actividad neurológica vinculada al conocimiento del uso digital varía de tareas de agarre a tareas de uso funcional de objetos y que la zona cortical relacionada con este último caso coincide con zonas del córtex que, como se expondrá en los sucesivos apartados de esta revisión, habitualmente se señalan como sustrato neurológico del pensamiento numérico (Buxbaum, Kyle, Tang y Detre, 2006).

Por otro lado también se ha puesto de manifiesto la estrecha relación que parece existir entre la gnosis digital y la representación numérica. Así, el conocimiento de los dedos se ha descrito como precursor específico de habilidades numéricas durante la infancia, pronosticando la evolución de éstas incluso con más exactitud que los test de inteligencia (Noël, 2005). De esta forma, la mejora en el propio conocimiento digital ha sido utilizado con éxito para potenciar el aprendizaje matemático durante la infancia (Gracia-Bafalluy y Noël, 2008).

Por otro lado, la relación entre orientación espacial y procesamiento numérico se ha confirmado en el estudio del papel que la zona parietal del córtex parece jugar en la generación de conductas topológicamente organizadas.

Así, Loetscher, Bockisch y Brugger (2008) registran que el patrón de movimientos oculares involuntarios que se perciben en sujetos ocupados en tareas vinculadas con procesamiento numérico (tales como determinar el número intermedio de un par de cifras) se ajusta a un movimiento de búsqueda a lo largo de un espacio orientado de izquierda a derecha y relacionan estas variaciones oculares con cambios en el espacio atencional interno. En opinión de estos autores, existe una homología entre las representaciones internas de espacio y número que hace pesar que el concepto de espacio numérico es, realmente, más que una metáfora (Loetscher et al., 2008, p.1) y que parece concordar con las numerosas evidencias que apuntan el procesamiento común de números, atención espacial y respuesta motora en zonas parietal y frontal del córtex cerebral. 
Estudios sobre patologías y lesiones

El análisis de cómo alteraciones en la estructura o en el normal desarrollo del córtex cerebral condicionan la actividad matemática permite estudiar qué regiones están implicadas en el manejo de cantidades y números. Igualmente, facilita la investigación en torno a la cuestión de si se puede considerar alguna diferenciación anatómica y funcional entre las regiones de la corteza cerebral implicadas en la comprensión numérica y las correspondientes a otro tipo de habilidades como el lenguaje.

Estas investigaciones se han desarrollado, por un lado, en torno a las consecuencias que determinadas anomalías genéticas generan en el pensamiento numérico y, por otro lado, en el estudio de las repercusiones que las lesiones cerebrales tienen en el procesamiento numérico.

Atendiendo a las investigaciones sobre las consecuencias que anomalías genéticas generan en la cognición numérica, la mayoría de los trabajos apuntan a la relevancia que la región parietal tiene con el manejo de números.

En este sentido, el análisis de las capacidades aritméticas de sujetos que padecen el síndrome velocardiofacial (malformación congénita caracterizada, entre otros síntomas, por anomalías craneofaciales, cardiopatías, retraso psicomotor y de la capacidad de aprendizaje) ha registrado alteraciones en el normal desarrollo de la zona parietal que se correlacionan con un déficit en las capacidades numéricas (Barnea-Goraly, Eliez, Menon, Roland Bammer y Reiss, 2005). Más específicamente, se sugiere que las anomalías en el surco intraparietal (zona parietal) son la causa más probable de las insuficiencias en las capacidades numéricas asociadas a este síndrome (De Smedt, Swillen, Devriendt, Fryns, Verschaffel, Boets y Ghesquière, 2007).

Por otro lado, se ha investigado los patrones de activación de la zona cortical entre sujetos con síndrome de Turner y se ha encontrado que éstos difieren de los patrones que muestran individuos de desarrollo normal, hecho que se ha vinculado con las pérdidas de las capacidades aritméticas de los individuos con síndrome de Turner (Molko, Cachia, Rivière, Mangin, Bruandet, Le Bihan, Cohen, y Dehaene, 2003; Shelli, Kesler, Menon y Reiss, 2006). Molko et al. (2003) consideran que estas carencias se relacionan con un desarrollo anormal del surco intraparietal. 
Han sido estudiados los déficits relativos al pensamiento matemático que presentan sujetos que padecen el síndrome de $\mathrm{X}$ frágil. Estos individuos parecen mostrar un patrón de activación del córtex diferente a los individuos normales, pero en este caso la principal diferencia se centra en un descenso generalizado de la activación neuronal incluso en los casos en los que se incrementa la dificultad de las tareas a realizar (Hessl, Rivera y Reiss, 2004).

Otra línea de investigación, examina las consecuencias que las lesiones cerebrales tienen en el procesamiento numérico. Los estudios sobre pacientes con daño cerebral confirman el rol de región parietal en el procesamiento numérico y en la comparación de cantidades, revelando que daños en esta zona del córtex impiden el adecuado manejo de números (Dehaene and Cohen 1997; Woods, Mennemeier, Garcia-Rill, Meythaler, Mark, Jewel y Murphy, 2006).

Sobre este particular resultan especialmente interesantes el estudio de cómo distintos patrones de lesión cerebral influyen en la acalculia. En esta línea se han analizado, por un lado, lesiones en la zona parietal izquierda que afectan especialmente a la región del surco intraparietal (IPS) y, por otro, la atrofia del córtex a la altura del lóbulo temporal izquierdo que cursaba síntomas de demencia semántica (Lemer, Dehaene, Spelke y Cohen, 2003).

Los resultados de estas investigaciones apuntan a que las lesiones en el IPS se relacionan con dificultades para efectuar con éxito tareas sencillas de reconocimiento y comparación cuantitativa de números y cantidades pequeñas; así como, para realizar operaciones de resta. Sin embargo, estas lesiones no se vinculan con carencias en habilidades aritméticas de mayor sustrato lingüístico como la multiplicación y la escritura y lectura de números.

Por otro lado, la atrofia del córtex lóbulo temporal vinculada a demencia semántica, se relaciona con dificultades para la ejecución de tareas con componente lingüístico como las multiplicaciones, siendo relativamente independiente de habilidades numéricas elementales como manejo de cantidades simbólicas y no simbólicas (por ejemplo, comparaciones entre matrices de puntos) y tareas de sustracción.

En este mismo sentido de estudio de la posible diferenciación funcional y anatómica de zonas implicadas en el pensamiento matemático, se ha puesto de manifiesto que pacientes con degeneración cortico-basal con especial afección en la zona parietal derecha del córtex 
presentan un menoscabo significativo en el manejo de números con relación a tareas de representación y denominación de objetos. Sin embargo, pacientes con demencia semántica que se relaciona con deterioros en la zona temporal anterior izquierda, mostraban una mayor debilidad hacia tareas que requerían nombrar y representar objetos (Halpern, Glosser, Clark, Gee, Moore, Dennis, McMillan, Colcher, y Grossman, 2004).

Butterworth et al. (2001) también registran el caso de un paciente con demencia semántica y consiguiente atrofia en el lóbulo temporal izquierdo que presentaba un declive severo de habilidades cognitivas en general y lingüísticas en particular. Sin embargo, sus habilidades matemáticas estaban sorprendentemente bien conservadas.

Igualmente, Klessinger et al. (2007) han investigado la disociación entre las capacidades algebraicas y lingüísticas en una caso de un paciente que padecía afasia concluyendo que, a pesar de que las habilidades en el dominio del lenguaje de este paciente están fuertemente disminuidas, su capacidad para emplear símbolos matemáticos está, manifiestamente, bien conservada.

A partir de sus investigaciones tanto Halpern et al. (2004) como Butterworth et al. (2001) concluyen en subrayar la relativa independencia anatómica y funcional que debe caracterizar a los dominios neuronales vinculados a la representación de números de aquellos ligados a la memoria semántica.

Polk et al. (2001) llegó a la misma conclusión a partir del estudio de un paciente con una lesión en el lóbulo parietal izquierdo que le imposibilitaba ejecutar adecuadamente tareas vinculadas al empleo de números tales como nombrar números, manejar las horas, estimaciones y empleo de cifras arábigas. Sin embargo, la paciente demostraba una buena capacidad para el manejo de símbolos de otras categorías semánticas (no numéricas) y de magnitudes continuas.

Esta diferenciación anatómica y funcional entre comprensión numérica y habilidades lingüísticas ha sido analizada recientemente por Baldo y Dronkers (2006) en una amplia muestra de pacientes que habían sufrido algún tipo de accidente cerebrovascular. Estos autores detallan que aunque existe una correlación general entre las habilidades lingüísticas y las 
aritméticas en el conjunto de los pacientes examinados, un buen número de éstos manifestaban signos de disociación funcional entre ambas capacidades.

Estos autores vinculan las habilidades aritméticas al córtex parietal mientras que las habilidades lingüísticas expresan una dispersión mayor, aún con una cierta orientación hacia la región temporal superior.

En resumidas cuentas, las líneas de investigación presentadas vienen a apuntar que las facultades básicas relacionadas con el manejo de números aparecen vinculadas a la zona parietal del córtex cerebral y que el procesamiento básico de estas capacidades podría ser relativamente independiente de otras zonas de la corteza cerebral vinculadas con las destrezas lingüísticas.

\section{Estudios de imagen cerebral}

Desarrollar un ámbito de conocimiento que se ocupe de la relación entre conducta y cerebro es una pretensión clásica dentro del estudio del comportamiento humano. Ya a finales del XIX James William (1842-†1910) enfatizaba en su trabajo The Principles of Psychology la necesidad de una ciencia de la mente que permitiera examinar los componentes básicos del comportamiento y las funciones de los elementos del cerebro. En palabras del autor, "una ciencia de la relación entre la mente y el cerebro debe mostrar cómo los ingredientes básicos del primero corresponden a las funciones elementales del segundo" (William, 1890).

Efectivamente superar la brecha entre la descripción de las conductas y los procesos neuronales subyacentes parece ser un reto permanente tanto en el ámbito de la psicología como en el de las neurociencias y para este fin, desde la década de los 70 del siglo pasado, se han ido desarrollando distintas técnicas que permiten monitorear las funciones cerebrales de manera, cada vez, más segura, detallada y cuantitativa.

\section{Aspectos metodológicos}

El desarrollo de las modernas técnicas de imagen cerebral comenzó a través de la tomografía computerizada de rayos X. Un procedimiento que abrió paso a técnicas más moder- 
nas como la tomografía por emisión de positrones (PET) y la resonancia magnética (MRI) (Raichle, 2003).

Dentro de las metodologías de imagen cerebral, existen dos tipos de categorías diferenciadas, las imágenes estructuradas y las funcionales. Las primeras se refieren al estudio de las partes y capas del cerebro, así como el diagnostico de afecciones morfológicas intracraneales y daños cerebrales. La segunda se refiere al estudio de los cambios que determinadas actividades cognitivas deliberadamente provocadas generan en el flujo sanguíneo de los capilares cerebrales y dentro de este grupo se consideran fundamentalmente tres técnicas, PET, fMRI y rTMS.

Dado que los estudios que relacionan estas técnicas de imagen cerebral con el pensamiento matemático se refieren, fundamentalmente, a este segundo apartado de técnicas, en lo sucesivo se describirán éstas como base para la comprensión de cómo trabaja el cerebro mientras manipula números.

\section{PET}

La tomografía por emisión de positrones, PET (siglas en inglés de Positron Emission Tomography), es una técnica de diagnóstico que consiste en obtener imágenes basadas en la detección de fotones gamma resultado de la destrucción de positrones provenientes de un radioisótopo.

El radioisótopo se suministra a través de una inyección o mediante inhalación y, con frecuencia, se utiliza el isótopo flúor-18, capaz de unirse a la glucosa para obtener el trazador 18-flúor-desoxi-glucosa. De este modo se logra una glucosa detectable mediante la emisión de señal radiactiva (Massardo et al., 2007). La causa de emplear glucosa es el elevado metabolismo glucídico que caracteriza al cerebro. Este órgano, aún no representando más que un $2 \%$ del peso corporal, consume un $20 \%$ del gasto calórico total del organismo (Raichle, 2003). Otros marcadores usados para la técnica de PET son isotopos del oxígeno y del nitrógeno.

A través de esta técnica no se evalúa la morfología de los tejidos, sino su nivel metabólico y los flujos sanguíneos preferentes y, consecuentemente, es útil para detectar áreas 
cerebrales involucradas en el procesamiento cognitivo. En este sentido, ha sido empleado con éxito en el estudio de zonas cerebrales involucradas en el pensamiento numérico (Thiouxa, Pesentia, Costesb, De Volderc y Serona, 2005; Pesenti, Thioux, Seron \& De Volder, 2000).

\section{$f M R I$}

La técnica denominada functional magnetic resonance imaging $(f M R I)$ es un tipo de neuroimagen que tiene su precursor en la tecnología de la resonancia magnética $(M R I)$. La característica fundamental de la resonancia magnética es que es una metodología que permite obtener imágenes del organismo de forma no invasiva, sin emitir radiación ionizante y en cualquier plano del espacio. Se fundamenta en propiedades magnéticas que poseen los núcleos atómicos, de forma que los campos de éstos son susceptibles de ser orientados en la dirección de un campo magnético externo. El fundamento de la técnica consiste en analizar la energía que liberan los núcleos de los átomos de hidrógeno de la molécula de agua en forma de ondas de radio una vez que los protones vuelven a su situación inicial.

Una de las principales diferencias de la $M R I$ con las técnicas de tomografía por emisión de positrones, se relaciona con el hecho de que la resonancia magnética analiza estructuras y no el nivel metabólico de los tejidos. La $f M R I$, por el contrario, mide, específicamente, a través de resonancia magnética, la respuesta hemodinámica de la actividad neuronal y, en consecuencia, este procedimiento arroja valoraciones funcionales de los tejidos analizados.

El fundamento de esta técnica esta vinculado al incremento metabólico de las redes neuronales implicadas en procesos cognitivos y que tienen como consecuencia el incremento del flujo sanguíneo en la zona neuronalmente activa y cambios en las concentraciones de oxihemoglobina y desoxihemoglobina. La oxihemoglobina es diamagnética y su influencia en el campo magnético es prácticamente despreciable. Sin embargo, la desoxihemoglobina es paramagnética lo que causa heterogeneidad en el campo magnético (Le Bihanl, 2007).

En regiones neuronales activas, el incremento de flujo sanguíneo implica un incremento de la concentración de oxihemoglobina cambiando las características de la relajación magnética. La resonancia magnética resulta ser sensible a estos cambios en la molécula de hemoglobina por lo que a través de esta técnica se pueden evaluar cambios en la actividad neuronal. 
Esta metodología ha sido empleada en una amplia variedad de investigaciones. De entre estas destacan aquellas que han intentado escudriñar la existencia de redes neuronales responsables del pensamiento aritmético aproximativo y de la estimación (Piazza, Pinel, Le Bihan y Dehaene, 2007) por la importancia que éstas tienen para avanzar en la comprensión de las representaciones numéricas pre-verbales.

Estas técnicas de imagen cerebral funcional son útiles no sólo para esclarecer que zonas son responsables de funciones cognitivas, sino, también para examinar cómo variables como la edad, la variación cultural o la interindividual y la experiencia y el aprendizaje influyen en el patrón de actividad cerebral (Dowker, 2006).

$r T M S$

El hecho de no poder establecer relaciones causales entre determinadas zonas cerebrales y capacidades para la realización de tareas precisas ha sido señalada como una importante limitación metodológica de los estudios realizados a partir de técnicas de resonancia magnética (Cappelletti et al., 2007).

Los estudios neurofisiológicos sobre pacientes con lesiones cerebrales han sido un metodología alternativa utilizada para establecer relaciones causales más certeras entre zonas cerebrales y tarea correspondientes. Sin embargo la plasticidad del cerebro para compensar insuficiencias funcionales así como la presencia en las lesiones de patologías adicionales e, igualmente, la falta de focalidad en las mismas, son inconvenientes que limitan las posibilidades de esta línea de investigación.

La estimulación magnética transcraneal ( $r T M S$, transcranial magnetic stimulation) es una metodología que pretende salvar la brecha que los estudios a través de neuroimágenes dejan entre correlación y causalidad ya que permite investigar los efectos de la modulación de la función cortical a través de una técnica no invasiva.

Básicamente la rTMS consiste en mantener un campo magnético fuera del cráneo el cual induce corrientes eléctricas en el cerebro las cuales alteran la actividad de redes neuronales. Las corrientes eléctricas inducidas, aunque breves y débiles, pueden despolarizar las membranas neuronales y descargarlas cuando sobrepasan determinados niveles (Cowey, 
2005). De esta manera se analizan las consecuencias que estas interferencias en la actividad neuronal generan en la conducta de sujetos neuronalmente sanos.

Con todo, actualmente no está claro cuál es el mecanismo a través del cual la estimulación magnética y la consiguiente inducción eléctrica interfieren en la actividad neuronal y cómo obstaculiza la acción cognitiva del individuo (Silvanto et al, 2007). En consecuencia, aunque esta técnica permite determinar si un determinada región cortical está o no implicada en una actividad cognitiva, no permite explicar la relación entre la zona neuronal y la actividad cognitiva considerada.

En cualquier caso rTMS representa una herramienta no invasora para experimentalmente inducir "lesiones virtuales" locales útil para el estudio de las funciones corticales del cerebro y está permitiendo a los investigadores examinar la contribución de diferentes áreas del córtex en la conducta y cognición, también en lo concerniente al pensamiento matemático (Cappelletti et al., 2007).

\section{Sistema neuroanatómico del pensamiento numérico}

Relativo a las cuestiones del papel que distintas zonas de la corteza cerebral pueden tener en la cognición numérica, así como de la diferenciación anatómica y funcional de las zonas implicadas en esta capacidad cognitiva, Dehaene y colaboradores (2003) han propuesto un modelo neuronal anatómicamente distinguible que sustentaría el procesamiento de la información numérica.

Este modelo, basado en investigaciones sobre imagen cerebral con $f M R I$, plantea la existencia de tres regiones de la corteza cerebral que se corresponden con otras tantas tareas precisas para el procesamiento numérico:

- La primera región se ubicaría en el segmento horizontal del surco intraparietal (hIPS); una zona del córtex que a través de las técnicas de imagen cerebral se ha visto que se activa de forma general durante la realización de todo tipo de tareas que se relacionan con el procesamiento numérico.

Se propone esta región cortical como responsable de la representación semántica de la magnitud propia de los números y de la representación no verbal de éstos a través de mapa de 
números o continuum numérico interno. De esta forma, permitiría entender lo que representa el tamaño numérico y de las relaciones de proximidad o lejanía entre los números.

- La segunda región se corresponde con el gyrus angular izquierdo $(l A G)$ que se ubica en la zona posterior e inferior del segmento horizontal del surco intraparietal del hemisferio izquierdo y se ha señalado como zona activa en determinadas tareas numéricas pero con funciones diferentes al hIPS.

En concreto la activación del $l A G$ no parece ligada a aspectos cuantitativos del procesamiento numérico sino que su activación parece estar más relacionada a tareas que exigen algún tipo de procesamiento verbal. Se propone que esta zona se vincula a representaciones verbales de los números y participaría en las tareas numéricas con mayor componente lingüístico del procesamiento de números como son determinadas actividades aritméticas como las multiplicaciones.

- La tercera región cerebral expuesta en el modelo se ubicaría en la zona posterior superior de ambos lóbulos parietales $(P S P L)$. Esta región está en la zona superior del hIPS y ocupa una posición mesial y superior con respecto a $l A G$. Aparece especialmente activa durante tareas de comparación entre números, cálculos aproximativos, resta de números y tareas de conteo.

Es una región con baja especificidad hacia el procesamiento numérico y más bien parece tener un importante papel en tareas que requieran visión espacial y en especial, en aquellas vinculadas a la movilidad de dedos, orientación y atención de la actividad visual, memoria espacial y visión espacial.

La activación de esta región cerebral parece justificable en el caso de tareas como el conteo, donde los sujetos deben atender a una sucesión de objetos y donde, en consecuencia, la orientación visual puede jugar un importante papel. Sin embargo parece más difícil de explicar su activación en operaciones numéricas de estimación, comparación y resta.

Sobre este particular se propone que la activación de esta región cortical sería necesaria para establecer relaciones espaciales adecuadas entre números dentro del continuum lineal que sostiene la representación interna de cantidades. De esta forma, la orientación espacial sería una capacidad necesaria para orientar los números en éste continuum y poder elaborar estimaciones y comparaciones entre ellos.

El papel que parece jugar el hIPS en la cognición numérica, ha sido confirmado, también, con otro tipo de metodologías. Así, Castelli et al. (2006) a través de un estudio mediante 
rTMS subrayan el papel de esta región del córtex en el procesamiento numérico tanto a través de representaciones simbólicas de los números (cifras arábigas) como no-simbólicas (matrices de puntos). Además los patrones de impedimento funcional tras la estimulación magnética transcraneal que este estudio presenta, revelan que éstos se hallan indefectiblemente unidos a la estimulación de la región del hIPS, mientras que no se han registrado efectos a partir de la estimulación de zonas anexas adyacentes como gyrus angular.

Los citados autores concluyen en la necesidad de considerar un mecanismo neural común para el procesamiento numérico de cantidades expresadas tanto simbólicamente como no simbólicamente. Knops et al. (2006) también a través de una metodología de investigación basada en rTMS coinciden en señalar el rol del hIPS en el procesamiento del significado cuantitativo de los números a la vez que encuentran un patrón diferencial entre varones y mujeres.

Recientes investigaciones, sin embargo, parecen apuntar que el papel del hIPS podría adentrarse más allá del procesamiento numérico situándose en el espacio del procesamiento del conocimiento abstracto ya que también ha sido expuesto su papel en la organización ordinal de series no numéricas (Fias et al., 2007).

Por otro lado, tal y como proponen Dehaene y colaboradores (2003), otras zonas del córtex también parecen tomar parte en el procesamiento de cantidades numéricas. En este sentido Rusconi y colaboradores (2005) encontraban que rTMS sobre angular gyrus izquierdo (lAG) obstaculizaba el procesamiento de tareas relativas a números pero en otro sentido al apuntado por el modelo de modelo de Dehaene, ya que, en este caso, se entorpecían las tareas numéricas basadas en manipulación con dedos. De todas maneras, otros trabajos muestran el relevante valor de AG en el procesamiento de habilidades lingüísticas (Stippich y al., 2007), en la línea apuntada por Dehaene et al. (2003).

Un aspecto que parece controvertido en el estado actual de las investigaciones parece ser el diferente papel que puede jugar el IPS derecho e izquierdo en el procesamiento numérico. Sobre este particular parece afianzarse la idea de que el IPS derecho tiene un papel relevante en el procesamiento de características continuas de los estímulos (Cappelletti, 2007) y que es una zona crítica para el procesamiento de magnitudes relativas al espacio, tiempo, tamaño y valor numérico. También Cohen et al. (2007) en un estudio sobre acalculia sostiene el 
rol del IPS derecho en el procesamiento de magnitudes. Por el contrario, el papel del IPS izquierdo parece más relacionado con el procesamiento simbólico y no simbólico de los números (Cappelletti, 2007).

\section{Cognición numérica comparada}

Aunque las investigaciones relativas a la sensibilidad de los animales para percibir aspectos cuantitativos pueden resultar realmente sorprendentes, lo cierto es que existe una vasta tradición de investigación en este campo que abarca más de 100 años (Jordan y Brannon, 2006).

Tal vez sean los pioneros trabajos del zoólogo alemán Otto Koehler (1889-†1974) los que dieron inicio a este ámbito de investigación a partir de sus experimentos con aves a través de los cuales logró establecer los límites superiores de la competencia numéricos de distintas especies: 5 para las palomas, 6 para los periquitos y 7 para los cuervos, loros y urracas (Hassenstein, 1974).

Desde entonces son numerosas las publicaciones en las que se dan cuenta de las capacidades numéricas de un amplio abanico de especies animales: peces (Agrillo, Dadda y Bisazza, 2007), aves (Lyon, 2003; Rugani, Regolin y Vallortigara 2007), roedores (Breukelaar y Dalrymple-Alford, 1998; Church y Meck, 1984), perros, (Ward y Smuts, 2007) cetáceos (Kilian, von Fersen y Gunturkun, 2005) y por supuesto, primates no-humanos (Beran, 2007; Cantlon y Brannon, 2007; Jordan et al., 2006; Van Marle, Aw, McCrink y Santos, 2006).

El hecho de constatar que la capacidad de entresacar consecuencias cuantitativas de los estímulos no es una habilidad cognitiva exclusivamente humana, es, en sí misma, una consecuencia científica de primer orden que sugiere interesantes preguntas sobre el origen filogenético de esta destreza y sobre su posible valor adaptativo (Díaz, 2006).

Sin embargo, actualmente las cuestiones de mayor interés sobre la cognición numérica animal son las referidas a dos aspectos: (a) conocer si la cognición numérica animal es un artificio de laboratorio y (b) estudiar los puntos en común que la cognición animal guarda con la humana. 
Sobre la primera de las cuestiones, el supuesto inicial que guió la investigación sobre cognición numérica en animales partía de la idea de que, dado que la noción de número es una abstracción no natural, los animales únicamente podía acceder a ésta a través del adiestramiento y, en consecuencia, no era una destreza que los animales manifestaran espontáneamente. En la base de este supuesto se sitúa la observación, bien documentada de que la conducta animal ligada a una comprensión numérica está fuertemente vinculada a la existencia de entornos ricos en estímulos y a la presencia persistente de recompensas para reforzar dicha conducta.

Los recientes resultados con primates no-humanos de Cantlon y Brannon (2007) relativos a esta cuestión son, empero, concluyentes al encontrar que animales sin entrenamiento previo, manifiestan conductas coherentes con una representación numérica cuando la razón de las cantidades era suficientemente discriminante. Por otro lado, registran, también, que la tendencia a atender a consideraciones numéricas de los estímulos es mayor que la que expresada hacia otro tipo de variables.

Concluyen estos investigadores que sus resultados permiten “...sostener el argumento de que monos y otros animales representan de forma espontánea valores numéricos. En consecuencia, debe rechazarse el argumento de que la cognición numérica de animales no humanos es una consecuencia del adiestramiento intensivo del laboratorio" (Cantlon y Brannon; 2007, p.40).

Flombaum, Junge y Hauser (2005) a través de un trabajo que adaptaba la metodología empleado por Wynn (1992a) con bebés, llegan a conclusiones similares. En concreto, estos autores demostraron que primates sin entrenamiento previo manifestaban, en el caso de transgresión de expectativas vinculadas a acciones de adición y sustracción de objetos, conductas atencionales significativamente diferentes con relación al comportamiento que mostraban frente a sucesos en los que se cumplían las expectativas aritméticas.

Esta investigación sugiere no sólo que los primates no-humanos son capaces de representar números y que son sensibles a variaciones aritméticas de colecciones sino que, además, poseen estas capacidades espontáneamente y que es posible que la noción de "número" sea una característica inherente al modo en que los primates no-humanos perciben los objetos (o conjuntos de objetos) también, en sus ambientes naturales (Cantlon, y Brannon, 2007). 
Una segunda cuestión es la consideración de qué puntos en común pueden tener humanos y primates no-humanos con relación a la representación numérica. Sobre este aspecto, parece existir una relativa unanimidad en torno a la idea de que tanto bebés (y humanos adultos) como primates parecen emplear dos sistemas de manejo numérico; uno vinculado a la representación precisa de cantidades inferiores a 4 objetos y otro sistema para la estimación aproximada de cantidades mayores (Flombaum et al., 2005).

Por otro lado, humanos y primates no-humanos muestran el mismo ajuste a la ley de Weber en tareas de discriminación estímulos numéricos no simbólicos, de forma que la discriminación de dos cantidades depende del radio entre ambas y no de los valores absolutos (Brannon, 2006; Cantlon et al., 2007; Flombaum et al., 2005).

De esta manera, tanto animales como humanos discriminamos con mayor rapidez y precisión matrices de puntos cuyo radio sea de 1:2 (por ejemplo, 8 versus 16) que matrices de radio 2:3 (4 versus 6) aunque los humanos muestran una mayor precisión, aún a costa de ejecutar las comparaciones más despacio (Cantlon et al., 2007). Finalmente, la representación de cantidades que primates no-humanos demuestran tener es, al igual que se ha comprobado en las investigaciones con bebes, independiente de la naturaleza del estímulo pudiéndose comprobar que tanto señales luminosas como auditivas son susceptibles de generar estas representaciones.

Es evidente que ni animales ni bebés pueden alcanzar los niveles de abstracción matemática que caracteriza al pensamiento humano adulto. Sin embargo, los datos presentados permiten pensar que los fundamentos de las habilidades matemáticas de humanos adultos se encuentren tanto en su historia evolutiva como en su desarrollo lo que llevaría a considerar que dentro del pensamiento matemático no sólo debe tenerse en cuenta el conocimiento matemático desarrollado a través del lenguaje e inmerso en una cultura, sino también, aquel sistema de representación numérica pre-lingüístico consecuencia de nuestra herencia filogenética (Brannon, 2006) 


\section{Cognición numérica desde la perspectiva de la psicología del desarrollo}

Los distintos paradigmas vinculados a la comprensión del desarrollo humano, tradicionalmente, han girado en torno a dos concepciones diferenciadas respecto al papel que el sujeto tiene sobre su propio desarrollo: o bien las estructuras cognitivas se desarrollan conforme a elementos precursores independientes, por lo menos en origen, de la propia acción del sujeto o bien, por otro lado, existen factores exógenos a las estructuras cognitivas de los individuos que determinan la evolución de las mismas.

Partir de uno u otro presupuesto teórico no puede ser considerado como un hecho baladí ya que, como se expondrá a continuación, el tipo de modelo que se adopte condicionará la metodología y la interpretación de los datos relativos al origen del pensamiento numérico. Retrotrayéndose al que se ha venido en considerar el periodo de expansión de la psicología del desarrollo y que viene a situarse a partir de la segunda mitad del siglo XX (Trigales y Gallardo, 2004), aparece la figura de Jean Piaget como uno de los primeros referentes indispensables para entender nuestra actual comprensión de cómo los seres humanos elaboramos la noción de número.

Desde la perspectiva piagetana (Piaget, 1980) se enfatiza el papel activo que el sujeto tiene en su propia evolución cognitiva. La acción es el elemento clave del cambio y la exploración y el descubrimiento son el estimulo que impulsa el desarrollo. A través de éstas, el sujeto desarrolla esquemas, inicialmente motores y posteriormente en forma de pensamiento, que le permiten una determinada comprensión de su entorno. Sin embargo, la estructura cognitiva actual no será definitiva en la medida que el sujeto durante la actividad exploratoria encuentre sucesos cuyas consecuencias no se ajusten a lo esperable. Surgirá la necesidad de modelar nuevamente les esquemas previos y, en consecuencia, surge una oportunidad para el desarrollo.

Es bien sabido que Piaget conceptualizó el desarrollo en etapas, en fases diferenciadas, de forma que desde el nacimiento a la adolescencia las características del pensamiento humano van transformándose a lo largo de cuatro etapas evolutivas que, como veremos posteriormente, determinarán las posibilidades de comprensión del concepto de número.

Dentro de este ámbito de énfasis de los factores exógenos a las estructuras cognitivas que incentivan el desarrollo, a partir de la década de los 70 se revindica la idea de que el desa- 
rrollo del ser humano no puede ser entendido sin considerar el contexto en el que éste se produce. Desde de las ideas de Lev Vygotsky (Garcia, 2005), se comienza a considerar el papel que la interacción social puede jugar en el desarrollo. Muy especialmente se subraya el lenguaje como medio a través del cual las personas interiorizan instrumentos y estrategias psicológicas que harán posible la representación y reflexión sobre la realidad y, en último término, la aprehensión de los elementos culturales característicos del entorno en el cual se desarrolla.

Estos planteamientos evolucionaron a partir de los años 80 en la corriente socioconstructivista, la cual destaca el papel activo de las personas como constructores activos de su propio desarrollo personal pero señalando, precisamente, los procesos interpersonales (intercambios sociales tales como el dialogo, la conversación y la negociación) como origen de los procesos superiores del pensamiento. Los entornos de aprendizaje son, desde este punto de vista, dominios privilegiados para la promoción del desarrollo ya que es precisamente en estos contextos (como la escuela, los grupos de educación no formal, etc.) donde el individuo encuentra la variedad y calidad de intercambios sociales (con adultos y con iguales) que pueden optimizar sus posibilidades de desarrollo.

Frente a estos paradigmas se sitúan aquellos que destacan la existencia de estructuras cognitivas precedentes que guían el propio desarrollo y que, cuando menos en origen, resultarían independientes tanto del contexto como de las acciones que el propio individuo pueda ejercer sobre el entorno. Dentro de esta perspectiva estarían fundamentalmente las teorías etológicas y las vinculadas al procesamiento de la información.

Las primeras parten de la idea de que el ser humano nace con una predisposición hacia determinadas conductas cuyos precursores pueden ser vistos también, en los animales. El desarrollo del ser humano estaría, en consecuencia, predeterminado por sus condicionantes genéticos y la influencia del entorno se circunscribiría a matizar dicho desarrollo (Blas, Gutiérrez y Bartolomé, 2005). Dentro de este contexto teórico puede ubicarse los estudios de pensamiento matemático comparado presentados en el apartado anterior, los cuales, en gran medida, comparten la siguiente idea latente: los rudimentos de la comprensión cuantitativa son una destreza evolutivamente seleccionada que genera algún tipo de beneficio adaptativo al organismo y de una insoslayable impronta genética. 
A partir de la década de los 80 surge como analogía del funcionamiento de los ordenadores, la conocida perspectiva del procesamiento de la información la cual ha influido decididamente la actual concepción de la comprensión numérica. Este paradigma parte de la idea de que los seres humanos disponemos de un sistema cognitivo susceptible de ser estudiado a partir de los elementos funcionales que lo componen (memoria, percepción, razonamiento,...) y que, en esencia, estos módulos funcionales son los mismo a lo largo del desarrollo. En consecuencia, dicho desarrollo es concebido, no como un paso de etapas, sino como una transformación continua, orientada a mejorar la eficacia de sus elementos funcionales que conforman el sistema cognitivo de la persona.

Una consecuencia de esta perspectiva fue la reacción a los postulados piagetanos desde una perspectiva innatista, la cual fue liderada por el funcionalismo de Jerry Fodor (1983). Este autor aboga por considerar el desarrollo como consecuencia del despliegue de sistemas o módulos de conocimiento específico (como el lingüístico, el matemático,...) que, por otro lado, son suministrados por la propia herencia genética.

Piaget y la comprensión de número.

Piaget como epistemólogo, se intereso por el origen del conocimiento e intentó aunar la perspectiva empirista que subraya el mundo exterior al individuo como fuente de conocimiento con el punto de vista racionalista que señala que la fuente de conocimiento no es la experiencia externa sino las capacidades racionales del individuo.

De esta manera Piaget admite que cierto tipo de conocimiento, como aquél relacionado con la comprensión del mundo físico y el vinculado a las convenciones sociales, tiene un principio, fundamentalmente, externo al propio sujeto. Sin embargo Piaget, igualmente, apunta otro tipo de conocimiento cuya raíz no puede situarse fuera de la propia mente. Este tipo de conocimiento surge a partir del establecimiento de relaciones entre objetos y su origen debe situarse en la propia acción reflexiva que el individuo ejerce sobre su experiencia con el mundo exterior. Esta abstracción reflexiva se diferenciada de la abstracción empírica vinculada únicamente con la información perceptible (Kamii, 1988).

Las características y naturaleza de estos tipos de conocimientos aparece bien ejemplificados en los materiales que Kamii y colaboradores (Kamii, Rummelsburg y Kari, 2005) uti- 
lizan para impulsar las capacidades lógicas y matemáticas de niños como los palillos chinos o mikado.

Este juego se inicia cuando tras dejar caer los palillos al azar y a lo largo del mismo,

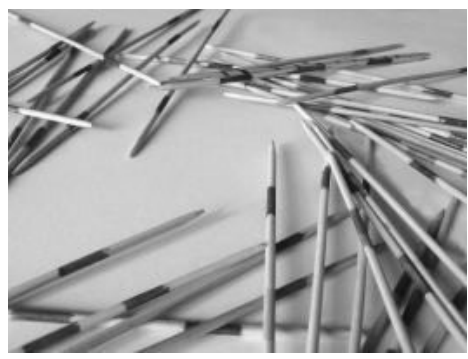
los jugadores intentarán coger el mayor número posible de palillos sin mover otros. En esta actividad el conocimiento físico está relacionado con las características propias de los objetos implicados, su tamaño, su peso, sus dimensiones, su color, etc. El conocimiento de las reglas del juego o de las palabras utilizadas para contar serían, por otro lado, ejemplos del conocimiento social. La causa de ambos tipos de conocimiento sería de naturaleza externa al individuo, situado bien en el mundo físico, bien en el contexto social.

Sin embargo, puede considerarse que dos palillos sean similares por sus dimensiones o diferentes por sus colores. En este caso, las consideraciones de semejanza o diferencia son un tipo de relación entre los objetos que la propia persona crea y cuyo origen se sitúa en la propia mente del individuo.

El conocimiento lógico-matemático pertenece, desde la perspectiva piagetana, a este último tipo de conocimiento el cual no puede inferirse directamente de la realidad sino que es consecuencia de las capacidades reflexivas del individuo para elaborar relaciones internas entre objetos. En este mismo sentido, tres objetos pueden ser observados pero no el númeroconcepto "tres" que es un conocimiento lógico-matemático a partir del cual se pueden elaborar relaciones de orden superior tales como $3+2=5$.

Piaget establece 5 aspectos del conocimiento lógico-matemático. Siguiendo con el material de los palillos chinos, en el momento de decidir que palillo coger el niño debe clasificar los palillos bajo el criterio de si tocan o no a otros palillos. Una vez que han sido agrupados todos los palillos que no tocan a otros, se deberán considerar los palillos que tienen un menor contacto con otros, estableciendo de esta manera una serie o sucesión desde los palillos más fáciles de tomar a los más difíciles. A partir de esta serie deben establecer un orden temporal para coger los palillos estableciendo una jerarquía o prioridad de palillos en el tiempo. Por otro lado, cuando deciden tomar preferentemente un palillo situado en la parte superior antes que otro situado debajo están estableciendo una relación espacial entre los objetos. Final- 
mente, cuando el juego termina se deben establecer entre los conjuntos de palillos de cada jugador, una relación numérica para determinar el ganador.

Piaget considera que los tres tipos de conocimientos expuestos (el físico, el convencional y el lógico-matemático) aparecen indiferenciados hasta la edad de 5-6 años y a partir de esta edad comienzan a distinguirse las distintas piezas de esta red de conocimiento.

El primero de ellos es la conservación de número. Éste es considerado el primer paso en la construcción del significado numérico, justo a partir de los 6 años (Piaget y Szeminska, 1965) y se refiere a la capacidad de abstraerse de las transformaciones de apariencia que pueden sufrir los conjuntos

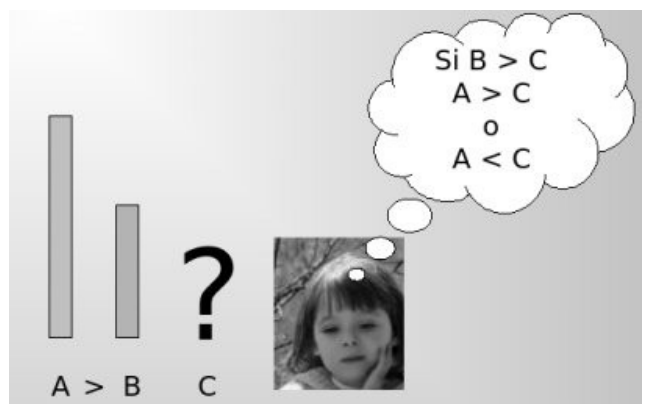
durante el conteo de objetos. De esta forma los niños podrán identificar conjuntos de un mismo cardinal como idénticos en cuanto al número de elementos, a pesar de que ambos conjuntos difieran en la distribución de los objetos que los componen.

Por otro lado, entre los 7 años se construyen otros dos tipo de relación mental señalados como por Piaget como indispensables en la comprensión numérica: la seriación y la clasificación (Inhelder y Piaget, 1964):

- $\quad$ La seriación es la habilidad para establecer comparaciones entre los elementos de un conjunto y ordenarlos de forma creciente o decreciente según sus diferencias.

De esta forma, el sujeto será capaz, por un lado, de deducir relaciones entre objetos que no se comparan directamente (transitividad) y, por otro lado, de concebir relaciones inversas entre elementos del conjunto de forma que pueda concluir que un objeto puede ser mayor que el anterior y, a su vez, menor que el siguiente (reversibilidad o capacidad para imaginar la acción contraria).

- La clasificación es la destreza cognitiva que permite establecer vínculos de semejanza, diferencia y pertenencia a conjuntos (relación entre un objeto y la clase a la que pertenece) y por otro parte, coordinar adecuadamente de las relaciones de inclusión entre una subclase a la que pertenece un objeto y la clase de la que forma parte (Labinowicz, 1986). 
A partir de estas consideraciones sobre el desarrollo de las habilidades lógicas de los niños, Piaget concluyó que la comprensión de la noción de número no es posible sin la aprehensión de estos fundamentos lógicos que permiten dar sentido a la acción de contar. La comprensión de la secuencia numérica es considerada una consecuencia de la síntesis de las capacidades de clasificación y seriación de modo que las capacidades aritméticas resultan de la generalización de las operaciones lógicas (Alonso et al., 2001; Bryant, 1996; Butterworth, 2005); lo que no resulta evidente hasta que los niños superan la etapa pre-operacional entre los 6 o 7 años.

\section{El paradigma del procesamiento de la información la comprensión de número}

Tal y como se ha expuesto, la perspectiva cognitiva del desarrollo humano y del proceso de elaboración del significado numérico personificada en Piaget entiende la interacción individuo-medio como germen del desarrollo. De esta manera se subraya el quehacer del propio sujeto como constructor del conocimiento a través de la acción y la experiencia sobre el medio. La comprensión de número es, en consecuencia, un proceso constructivo fundamentado en bases lógicas que ni preexisten al propio conocimiento (no pueden ser halladas antes de ser elaboradas) ni pueden ser directamente enseñadas (por lo que el papel del educador está esencialmente relacionado con la creación de medios ricos en experiencias que potencien las posibilidades de formación de nuevos esquemas representacionales de la realidad).

A partir de la segunda mitad del siglo XX, y partiendo de la necesidad de conocer con mayor precisión las habilidades y limitaciones cognitivas, aparece un nuevo enfoque dentro de la psicología cognitiva muy vinculado a los adelantos tecnológicos y en particular, a las innovaciones informáticas. Esta nueva perspectiva que se ha venido en denominar teoría del procesamiento de la información, supuso una forma novedosa de abordar el estudio de las funciones mentales tales como la memoria, la atención, las representaciones mentales y, como no, también, la comprensión matemática.

Un elemento central del paradigma del procesamiento de la información, se refiere al hecho de que el desarrollo, desde está perspectiva, no se vincula con variaciones sustanciales de la estructura cognitiva sino que, más bien, se relaciona con una progresiva adecuación en la manera de manejar la información. De esta forma se entiende que el procesamiento cognitivo es esencialmente el mismo a todas las edades (en cuanto a los mecanismos cognitivos 
empleados, por ejemplo, los receptores sensoriales, la memoria de trabajo o las capacidades atencionales) y el desarrollo está relacionado con la mejora funcional del sistema de forma que el individuo va adecuando los procesos de captación de estímulos, la capacidad de analizarlos y asociarlos, la información acumulada, las estrategias de aprendizaje, etc. (Blas et al., 2005; Trianes et al., 2004).

Este nuevo paradigma ha tenido dos consecuencias fundamentales en la investigación del origen del pensamiento numérico.

Por un lado, ha suscitado un renovado interés hacia el estudio de las habilidades numéricas tempranas, poniendo en valor, por un lado, el fenómeno del conteo cuyas primeras manifestaciones son visibles a partir de los 18-20 meses y, por otro, la sensibilidad que prácticamente desde los primeros meses de vida los seres humanos demostramos ante variaciones cuantitativas de los estímulos.

La segunda consecuencia se relaciona con la discusión relativa a qué elementos del sistema cognitivo pueden ser considerados innatos y si entre éstos se encuentran las bases estructurales del pensamiento numérico.

A continuación se presentan los resultados y perspectivas que estas líneas de investigación han ofrecido. En primer lugar se presentará el modelo de aprehensión numérica en cuatro pasos principalmente con el fin de exponer una visión general de los mecanismos cognitivos que actualmente se han detectado en la investigación sobre el pensamiento numérico temprano. Seguidamente, se mostraran las averiguaciones que se han realizado en torno a las habilidades numéricas previas al desarrollo de las destrezas lingüísticas. En la parte final de esta sección se discutirá la controversia que existe en torno a la que posiblemente sea primera adquisición genuinamente matemática durante el desarrollo: el conteo.

\section{Modelo de aprehensión numérica}

Recientemente se ha propuesto un marco interpretativo para la comprensión de disfunciones de la comprensión numérica tales como la discalculia (Butterworth, 2005) que resulta útil, también, como modelo para la comprensión del desarrollo de las habilidades númerocogntivas tempranas. 


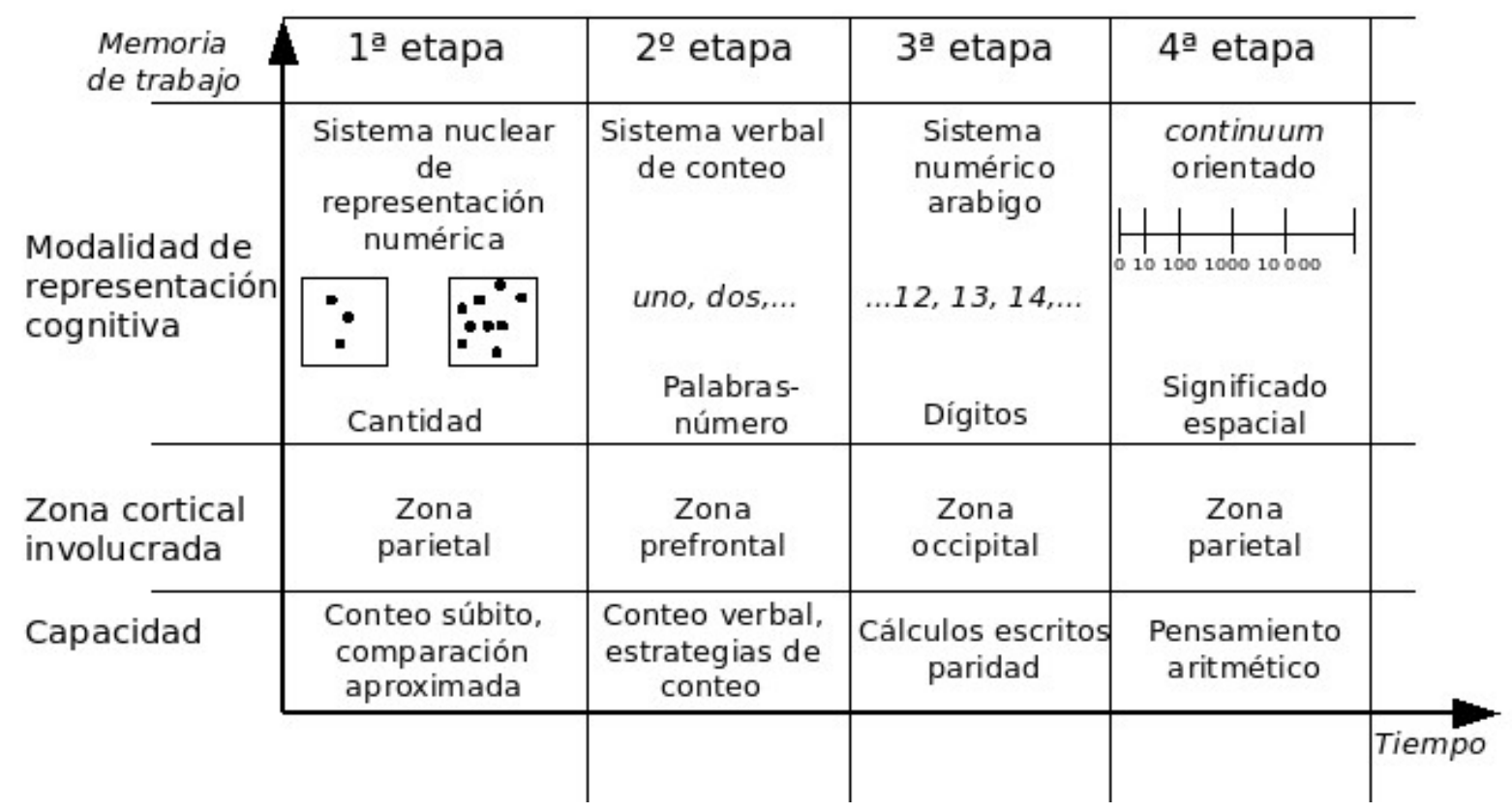

Figura 1. Ilustración del modelo en cuatro fases de von Aster y Shalev (2007).

Este modelo planteado por von Aster y Shalev (2007) postula el desarrollo de la representación numérica durante la primera infancia como un proceso gradual de adquisición de estrategias para la representación de cantidades que se manifiesta fases.

A continuación se muestran las cuatro periodos propuestos y después se dará cuenta de los datos neuropsicológicos que sustentan este modelo.

1. Desde los primeros meses de vida y antes de la exteriorización de las primeras manifestaciones lingüísticas, los individuos tendrían acceso a las primeras representaciones de cardinalidad a través de un sistema primigenio o nuclear. Se postula que a través de este sistema cognitivo (que como se explicará más adelante parece estar integrado por varios subsistemas) los seres humanos demostraríamos prácticamente desde el propio nacimiento sensibilidad hacia las características y variaciones cuantitativas de los estímulos.

Esta sensibilidad vinculada a la detención súbita y a la comparación de cantidades y se nos parece estrechamente ligada con la actividad parietal del córtex cerebral.

2. La aparición de las primeras manifestaciones de un sistema verbal de conteo, entre los 24 y 30 meses de edad, daría paso a la segunda fase del desarrollo de la comprensión numérica caracterizado, en primer lugar, por el progresivo uso de palabras-número y, posteriormente, por la aparición de las estrategias de enumeración. En este caso, esta habilidad im- 
plicaría zonas del córtex cerebral habitualmente más relacionadas con las capacidades verbales como el córtex prefrontal.

3. La utilización de símbolos escritos para referirse a los números (sistema arábigo de numeración) marca el comienzo de la tercera fase en la cual se inicia el aprendizaje de los dígitos, los cálculos escritos y determinadas características de éstos tales como el concepto de paridad.

4. Durante las fases 2 y 3 , se va elaborando la simbolización del número lo que es considerado un paso previo y necesario al desarrollo de la representación semántico-espacial de los números ilustrada a través del modelo de continuum orientado de izquierda a derecha y en el que cada número corresponde a un punto de la línea. Nuevamente, la zona parietal del córtex aparece especialmente vinculada a la formación de la representación espacial de los números.

La cuestión del origen del pensamiento numérico aparece especialmente influenciada por las investigaciones vinculadas a las dos primeras fases de este desarrollo. En este sentido, a continuación se presenta una revisión de la investigación en torno a las capacidades numéricas prelingüísticas y, por otro lado, al desarrollo de las habilidades para el conteo

\section{Habilidades numéricas prelingüísticas}

Coincidente con la propuesta expuesta, en los que se refiere a la primera de las etapas, Spelke y Kinzler (2007) plantean también, la existencia de un núcleo cognitivo primigenio constituido por un reducido número de subsistemas entre los que se encuentra el relativo a la representación numérica.

Estas representaciones cumplirían en opinión de estas investigadoras las características siguientes:

- Son imprecisas y esta falta de exactitud crece con el incremento del valor cardinal. Sin embargo la precisión mejora con el desarrollo. Por otro lado, resultan dependientes del radio de las cantidades de forma que la capacidad de discriminar conjuntos de diferente valor cardinal es sensible al ratio de las cantidades que se comparan. 
- Son abstractas dado que resultan independientes de la modalidad sensorial y, en consecuencia, pueden ser consecuencia del estímulo visual producido por una matriz de puntos, una secuencia de sonido o de una ordenación de acontecimientos.

- $\quad$ El número representado puede ser comparado y combinado por operaciones de adicción y sustracción.

Una detallada revisión de la investigación en torno a estas representaciones numéricas prelingüísticas permite catalogar tres tipos de representación cuyas características se ajustan a las antedichas por Spelke et al. (2007) (Barner, Thalwitz, Wood, Yang y Carey, 2007; Condry y Spelke, 2008; Feigenson et al., 2004; Le Corre y Carey, 2007):

1. Representación exacta de cantidades pequeñas, menores de cuatro elementos y que son posibles mediante la elaboración en la memoria de trabajo de modelos en los que cada objeto del conjunto es representado mediante un único símbolo mental (Le Corre y Carey, 2007, 2008). A través de estos modelos, pueden determinar equivalencias y comparaciones numéricas.

Las investigaciones dentro en torno a este tipo de representaciones se centran en estudiar la capacidad de los niños y niñas para "seguir" objetos que se muestran (galletas, bolas,...) y desaparecen y analizar sus respuestas atencionales o de búsqueda (Feigenson et al., 2004 presentan una revisión metodológica). En esta línea, Feron, Gentaz y Streri (2006) prueban que niños y niñas de 5 meses de edad son capaces de elaborar representaciones de conjuntos pequeños de objetos, memorizarlas y utilizarlas para realizar comparaciones independientes de la modalidad sensorial empleada para presentar los estímulos y de otras características de los objetos tales como la forma o el tamaño. Por su parte, Kobayashi, Hiraki, Mugitani y Hasegawa (2004) también demuestran la capacidad de niños y niñas de 5 meses para elaborar representaciones abstractas y amodales de colecciones pequeñas de objetos. Estos autores evidencian también, la sensibilidad de los bebes a variaciones aritméticas del número de objetos.

Feigenson y Carey (2005) registran igualmente la capacidad de diferenciar conjuntos de objetos de 1 vs. 2 ; 1 vs 3 y 2 vs 3 de niños a partir de los 10 meses. Con todo y que parece existir una cierto acuerdo en corno a las capacidades que para representar colecciones peque- 
ñas de objetos demuestran los recién nacidos, algunos trabajos (Xu, Spelke, Goddard, 2005) han presentado dentro de esta línea de investigación, resultados contrapuestos subrayando que las capacidades de discriminación numérica de colecciones pequeñas de objetos pudiera estar unida a cambios en variables continuas de los objetos (superficie total, brillo, longitud del contorno en formas bidimensionales y volumen total en el caso de objetos tridimensionales). En cualquier caso, la metodología empleada en el trabajo de Xu y colaboradores (2005) difiere sustancialmente de las investigaciones en este campo y pretende, esencialmente, determinar la existencia de dos sistemas de representación numérica, el correspondiente a este apartado y el vinculado a representación de cantidades aproximadas que se presenta a continuación.

2. Representación aproximada de cantidades a través de un sistema cognitivo que representa el valor cardinal como una magnitud continua proporcional a la cantidad de objetos. La investigación en torno a este sistema se centra en el estudio de la capacidad de los individuos (adultos humanos, bebes y animales, -Xu y Arriaga, 2007; Brannon, 2006, Barth et al., 2005-) para diferenciar colecciones numerosas de objetos sin recurrir, en su caso, al conteo. Una característica de este sistema de representación numérica es que la capacidad de discriminar conjuntos es dependiente del ratio de la mismas, de forma que, en el caso de los niños, a los 6 meses son capaces de diferencias cantidades de ratio 1:2 (4 vs $8 ; 8$ vs 16 y 16 vs 32) pero no cuando la razón es de 2:3 (4 vs 6; 8 vs 12 y 16 vs 24). Sin embargo, a partir de los 10 meses de edad sí son capaces de discernir ratios 2:3. En este sentido, la evolución de esta capacidad se supone vinculada al desarrollo (Le Corre, et al. 2007).

3. Finalmente, se puede distinguir un tercer sistema de representación numérica relaciona con la capacidad de distinguir lo singular de lo plural y que se situaría en la base del uso de los cuantificadores lingüísticos (uno, todos, muchos, algunos,...) (Le Corre, et al. 2007; 2008). Recientes estudios parecen demostrar que esta capacidad representacional está presente en primates no-humanos y en niñas a partir de los 14 meses de edad (Barner et al., 2007).

\section{El origen del número natural: la controversia sobre el conteo}

Resulta conocido por educadores e investigadores que los niños y niñas entre el segundo y tercer año de vida y con creciente habilidad utilizan palabras-número para tareas de conteo. Piaget, tal y como se ha explicado anteriormente, otorgó escaso valor a estas primeras 
manifestaciones numéricas ya que resultaban previas a la aprehensión de los fundamentos lógicos que permiten entender la noción de número.

Sin embargo, una serie de interesantes publicaciones los profesores Rochel Gelman y Randy Gallistel (Gallistel y Gelman, 1992; Gelman y Gallistel, 1978; Gelman y Gallistel, 2004) proponen una visión relativa al significado del conteo sustancialmente diferente, reorientando el núcleo de la cuestión del origen del pensamiento numérico hacia el estudio de las destrezas funcionales de los niños y alejándose del análisis del nivel de comprensión de las bases lógicas del concepto de número.

Estos autores proponen que el niño que es capaz de contar, realmente sí posee una representación del número natural, por lo menos, en la medida que sea capaz de demostrar la aprehensión de los siguientes principios de conteo: orden estable de las palabras conteo; cardinalidad, entendida como el significativo valor que la última palabra-número tiene para caracterizar la colección y, finalmente, correspondencia, en el sentido que todos los objetos de la colección deben ser contados y únicamente una vez (Bryant, 1996).

Su propuesta relativa a la ontogenia de la comprensión del concepto de número natural (Gallistel y Gelman, 1992; Gelman y Gallistel 1978) va, no obstante, más allá; proponiendo que el origen de los principios de conteo es, esencialmente, innato. Estos autores postulan que durante el aprendizaje del conteo se produce una asociación entre el sistema de representación numérica aproximado que representa las cantidades de una forma imprecisa y continua con los marcadores de posición que suponen las palabras conteo (Le Corre, 2007). En este sentido, desde la perspectiva de estos autores, las primeras palabras-conteo están cargadas de significado y no son meros aprendizajes memorísticos sino que se refieren a la primera representación explícita del número natural.

Sin embargo, frente a esta posición las investigadoras Mathieu Le Corre y Susan Carey $(2007 ; 2008)$ mantienen un perspectiva encontrada. A pesar de que también entienden que las representaciones numéricas aproximadas son ciertamente relevantes, no otorgan a éstas el papel primigenio en la formación del concepto de número. De hecho estas autoras proponen que las primeras palabras conteo aparecen como aprendizajes memorísticos que se van cargando de significado numérico en la medida que el niño va asociándolas con los resultados del sistema cognitivo de representaciones exacta de cantidades pequeñas. De hecho, Gallistel 
y colaboradores únicamente admiten la existencia del sistema de representación aproximada de cantidades como magnitud continua.

Detrás de esta controversia existe un profundo debate entre las posiciones nativistas y constructivistas de la adquisición de la habilidad del conteo. Sin embargo, son estas últimas las que está recibiendo un mayor apoyo, sobre todo a tenor de un hecho ampliamente registrado: existe un intervalo de un año desde que los niños y niñas comienzan a recitar las palabras conteo hasta que realmente las emplean como representación de números naturales.

Esta demora explicitada en los trabajo de Wynn (1992b) se refiera al hecho de que a través de tareas en las que se propone a los niños que determinen conjuntos de objetos (por ejemplo, pidiéndoles que ayuden a un títere o muñeco a alcanzar un determinado número de objetos) se han determinado dos fases:

- $\quad$ Fase de conjunto-conocedor: Durante esta etapa los niños y niñas son capaces de delimitar conjuntos de 1, 2 o 3 elementos. Durante este periodo la especificación de los conjuntos se realiza por conteo súbito, sin recurrir a las palabras-número. De hecho, suelen demostrar una falta del principio de cardinalidad y si se les propone contar los objetos, tampoco recurren a una orden estable de las palabras conteo. Esta fase suele durar en torno a un año y los niños van pasando de ser conjunto-uno-conocedores a dos-conocedores y tresconocedores en sucesivos periodos de 4-5 meses.

- $\quad$ Alrededor de los 3,5 años las niñas y niños alcanzan una nueva etapa, que se suele denominar cardinal-conocedor coincidente con el momento en que comienzan a demostrar su capacidad para seleccionar conjuntos de cuatro y más objetos. A partir de este momento, recurren a las palabras conteo con más frecuencia en tareas de numeración, especialmente para conjuntos de más de 4 elementos y respetan el principio de orden estable.

Precisamente en torno a esta constatación de que los niños suelen necesitar entre 12 a 15 meses para pasar de ser conjunto-uno-conocedores a cardinal-conocedor, surge una mayoritaria adhesión en torno a la idea de que este proceso no se ajusta al supuesto de innatismo de los principios de conteo y que, más bien al contrario, parece reflejar un proceso de elaboración cognitiva, en este caso, del significado de las palabras-conteo (Condry et al., 2008; Le Corre, Van de Walle, Brannon y Carey, 2006; Sarnecka, Kamenskaya, Yamana, Ogura y Yudovina, 2007). 
Los principios de conteo, en consecuencia, más que disposiciones inherentes serían consecuencia de un proceso de asignación de las primeras palabras-número ("uno", "dos" y "tres") a representaciones internas de colecciones de objetos creadas por los sistemas cognitivos vinculados a la representación exacta de cantidades pequeñas y a la distinción de singular y plural que, aunque de capacidad limitada, permiten al niño focalizar la atención en varios objetos simultáneamente y elaborar representaciones de un grupo de objetos (Le Corre et al., 2007; Le Corre et al., 2008).

Estas representaciones no serían, propiamente, representaciones del concepto de cardinalidad de una colección ya que este principio no aflorará hasta que el niño o niña comprenda que añadiendo un objeto a la representación de uno se consigue dos y que, a su vez, incorporando a este último otro objeto se logra tres (Condry et al., 2008). Sin embargo, estas representaciones sí resultarían útiles para relacionarlas con las palabras-número facilitando el primer peldaño de la acción de numeración

\section{Conclusiones y consideraciones didácticas}

A través de este escrito se ha mostrado que la cuestión del origen y desarrollo del pensamiento numérico dista de poder ser abordada desde una única perspectiva y que, dadas las diversas aportaciones que diferentes líneas de investigación con abordajes metodológicos particulares ofrecen, resulta indispensable un punto de vista ecléctico y multidisciplinar.

Precisamente, este repaso alrededor de las contribuciones de áreas de conocimiento como las neurociencias, los estudios comparados, los modelos cognitivos y la psicología experimental, a los que, seguramente, debería añadirse las investigaciones antropológicas y lingüísticas, dibuja un panorama multiparadigmático sobre la cuestión de cómo los seres humanos construimos la noción de número natural.

Parece razonable pensar de este panorama diverso debiera traducirse, también, en variedad de planteamientos para la acción didáctica y educativa.

Ciertamente partiendo del punto de vista piagetano, existe una tradición ampliamente reconocida que aborda la cuestión didáctica de la enseñanza de la noción de número a partir del desarrollo de las operaciones lógicas. 
En esta línea, numerosos autores (Kamii, Rummelsburg y Kari, 2005) y propuestas didácticas (Blas et al., 2005; Labinowicz, 1986; Ruesga, 2004) plantean el trabajo de aula a partir, esencialmente, de actividades tendentes a desarrollar los fundamentos lógicos de la noción de número. Desde esta perspectiva es baldía la enseñanza directa del concepto de número, por ejemplo, a través de rutinas, fichas o conteos directos. Más la contrario se potencia la formación de entornos ricos en experiencias con objetos que faciliten al niño y la niña la identificación de las cualidades de objetos proponiendo tareas de identificación de elementos con cualidades similares, de comparación de objetos a partir de características semejantes, de formación de agrupaciones espontáneas, etc.

En este tipo de propuestas son habituales las tareas en las que a partir de objetos manipulables, se proponen actividades de seriación (organizar elementos considerando la diferencia que hay entre ellos con respecto a una determinada variable para poder establecer una secuencia), clasificación (comparaciones entre objetos con relación a una variable y agrupación de aquellos que son semejantes), correspondencias (relaciones término a término entre colecciones de objetos) y operaciones (transformaciones y operaciones de unión y complementación) (Ruesga, 2004).

Huelga subrayar las indiscutible aportación que esta metodología ofrece al desarrollo cognitivo y numérico de los niños. Sin embargo, desde la perspectiva derivada de los modelos de desarrollo del pensamiento numérico vinculados al procesamiento de la información, se sostiene que las capacidades numéricas no son ajenas a niños y niñas menores de 6 años y se subraya que el aprendizaje de las palabras-número y su empleo en actividades de conteo son elementos claves de la aprehensión de la noción de número (Sarnecka et al., 2007). Este punto de vista ofrece aportaciones complementarias interesantes y permitiría justificar la utilidad, también, de diseños didácticos que, además de tareas relacionadas con capacidades lógicas, ofrezcan oportunidades para el uso directo de cifras y números a través de actividades que de forma directa inviten a la numeración y al empleo y aprendizaje de las palabras número y sus correspondientes símbolos a través de rutinas lingüísticas y elementos gráficos variados (Fernández, 2006; Klein, Starkey y Ramirez, 2003)

Por otro lado, desde el mundo de las neurociencias también aparecen sugerencias interesantes para la acción didáctica. Tal vez, la estrecha relación que a nivel neurológico se ha 
evidenciado entre pensamiento numérico y gnosis digital sea uno de las aportaciones más sugerentes.

Tal y como se ha expuesto a lo largo de este artículo, la conjetura de que existe una estrecha relación entre el conocimiento de los dedos y el pensamiento numérico tiene una larga historia que arranca desde las primeras descripciones que se hicieron a comienzos del siglo pasado del síndrome de Gerstmann (Alonso et al., 2001; Lebrun, 2005).

De hecho, se constata que la actividad neurológica vinculada al conocimiento digital está relacionada con el tipo de actividad de los dedos de forma que las tareas de uso funcional de objetos estimulan más frecuentemente zonas del córtex parietal que se destacan, igualmente, por su relación con la cognición numérica (Buxbaum et al., 2006).

Otra constatación importante es que el conocimiento digital predice de forma bastante selectiva el nivel de consecución de habilidades numéricas durante la etapa escolar (GraciaBafalluy et al., 2008; Noël, 2005) y que incluso, dada esta relación, el trabajo en el conocimiento y diferenciación de los dedos es un tipo de actividad complementaria eficaz para mejorar la competencia numérica (Gracia-Bafalluy et al., 2008; Kaufmann, 2008).

Finalmente, las neurociencias introducen también, un tema de gran incidencia educativa como es el relativo a las dificultades de aprendizaje y, más concretamente, al fenómeno de la discalculia.

Este desorden del aprendizaje puede afectar a un 5-6\% de la población escolar (Shalev, 2004) y se relaciona con una dificultad persistente en el proceso de adquisición de destrezas numéricas y aritméticas. Inicialmente ocasiona falta de comprensión del conteo y dificultades en el uso de éste para tareas de adicción simples. A medio plazo se vincula con un importante déficit para la memorización de eventos aritméticos (como las tablas de suma y multiplicación). Se discute también que esta deficiencia pueda afectar a niveles más básicos del aprendizaje numérico como son la representación interna de cantidades y la destreza para ligar cantidad con su representación simbólica.

Con relación a su origen, se relaciona con carencias neurológicas específicas y puede aparecer de forma aislada o vinculada a otros problemas de aprendizaje como la dislexia pero, 
en cualquier caso, la incidencia final en el sujeto depende de la adopción de medidas didácticas específicas (Willburger, Fussenegger, Moll, Wood y Landerl, 2008).

Sobre esta cuestión dos líneas de trabajo parecen relevantes. La primera se refiere al trabajo sistemático de comparación espacial entre números, de refuerzo de la conexión entre representaciones simbólicas y no-simbólicas de cantidades y de representación gráfica de hechos aritméticos con cantidades pequeñas (Wilson, Revkin, Cohen, Cohen y Dehaene, 2006). La segunda línea de trabajo nos devuelve, de nuevo, a la cuestión de la gnosis digital en el desarrollo de las habilidades aritméticas ya que el conteo y cálculo mediante los dedos facilita la representación mental del número, posiblemente favoreciendo el establecimiento de la relación entre la percepción de cantidades no simbólicas con la representación interna y abstracta de éstas, también en niños y niñas que padezcan discalculia (Kaufmann, 2008). 


\section{Referencias}

Agrillo, C.; Dadda, M. \& Bisazza, A. (2007). Quantity discrimination in female mosquitofish. Animal Cognition, 10 (1), 63-70.

Alonso, D. \& Fuentes, L. (2001). Mecanismos cerebrales del pensamiento matemático. Revista de Neurología, 33, 568-576.

Balasubramanian, V. (2006). Isolated agraphia:next term A cognitive-neuropsychological analysis. Brain and Language, 99, 23-25.

Baldo, J. \& Dronkers, N. (2006). Neural correlates of arithmetic and language comprehension: A common substrate?. Neuropsychologia, 45 (2), 229-35.

Barnea-Goraly N.; Eliez, S.; Menon, V.; Roland Bammer, R. \& Reiss, A. (2005). Arithmetic ability and parietal alterations: A diffusion tensor imaging study in Velocardiofacial syndrome. Cognitive Brain Research, 25 (3), 735-740.

Barner, D.; Thalwitz, D.; Wood, J.; Yang, S. \& Carey. S. (2007). On the relation between the acquisition of singular-plural morpho-syntax and the conceptual distinction between one and more than one. Developmental Science, 10 (3), 365-373.

Barth, H.; La Mont, K.; Lipton, J. \& Spelke, E.S. (2005). Abstract numbr and arithmetic in preschool children. PNAS, 102 (39), 14116-14121.

Blas, A.; Gutierrez, D. \& Bartolomé, R. (2005). Educación Infantil. Madrid: Mc Graw Hill.

Brannon, E. (2006). The representation of numerical magnitude. Current Opinion in Neurobiology, 16, 222-229.

Bryant, P. (1996). Mathematical Understanding in the Nursery School Years. En Learning and Teaching Mathematics. An International Perspective, Psychology Press, 53-67.

Beran, M. (2007). Rhesus monkeys (Macaca mulatta) enumerate large and small sequentially presented sets of items using analog numerical representations. Journal of Experimental Psychology: Animal Behavior Processes, 33 (1),42-54.

Berg, D.H. (2008). Working memory and arithmetic calculation in children: The contributory roles of processing speed, short-term memory, and reading. Journal of Experimental Child Psychology, 99 (4), 288-308.

Brannon, E. (2006). The representation of numerical magnitude. Current Opinion in Neurobiology, 16, 222-229. 
Breukelaar, J. \& Dalrymple-Alford, J. (1998). Timing ability and numerical competence in rats. Journal of Experimental Psychology. Animal Behavior Processes, 24, 84-97.

Butterworth, B., Cappelletti, M., \& Kopelman, M. (2001). Category specificity in reading and writing: the case of number words. Nat.Neurosci. 4, 784-786.

Butterworth B. (2005). The development of arithmetical abilities. Journal of Child Psychology and Psychiatry 46 (1), 3-18.

Buxbaum L.; Kyle K.; Tang K. \& Detre J. (2006). Neural substrates of knowledge of hand postures for object grasping and functional object use: Evidence from fMRI. Brain Research, 1117 (1), 175-185.

Campbell, J.I. \& Lynette, J. (2004). An Encoding-Complex Approach to Numerical Cognitionnext term in Chinese-English Bilinguals. Canadian Journal of Experimental Psychology, 58 (4), 229-244.

Campbell, J.I. \& Metcalfea, W.S. (2008). Arabic digit naming speed: Task context and redundancy gain. Cognition, 107 (1),218-237.

Cantlon, J. \& Brannon, E. (2007). How much does number matter to a monkey (Macaca mulatta)?. Journal of Experimental Psychology: Animal Behavior Processes 33 (1), $32-$ 41.

Cappelletti, M.; Barth, H.; Fregni, F.; Spelke, E. \& Pascual-Leone, A. (2007). rTMS over the intraparietal sulcus disrupts numerosity processing. Experimental Brain Research, 179 (4), 631-642.

Castelli, F.; Glaser, D. \& Butterworth, B. (2006) Discrete and analogue quantity processing in the parietal lobe: a functional MRI study. PNAS, 103, 4693-4698.

Church, R. \& Meck, W. (1984). The numerical attributes of stimuli. In Roitblat, Herbert, Thomas Bever \& Herbert Terrace (eds.), Animal cognition, 445-64. Hillsdale, NJ: Lawrence Erlbaum Associates.

Cohen, R.; Cohen, K.; Schumann, T.; Kaas, A.; Goebel, R.; Henik, A. y Sack, A. (2007) Virtual dyscalculia induced by parietal lobe TMS impairs automatic magnitude processing. Current Biology, 17 (8), 689-693. 
Cohen, R.; Lammertyn, J. \& Izard, V. (2008). Are numbers special? An overview of chronometric, neuroimaging, developmental and comparative studies of magnitude representation. Progress in Neurobiology (84), 132-147.

Condry, K.F. \& Spelke, E.S. (2008). The Development of Language and Abstract Concepts: The Case of Natural Number. Journal of Experimental Psychology: General, 137 (1), $22-38$

Corvera, N.; Doetjesb, J. \& Zwartsa, J. (2007). Linguistic perspectives on numerical expressions: Introduction. Lingua, 117 (5), 751-757.

Cowey, A. (2005) The Ferrier Lecture 2004: what can transcranial magnetic stimulation tell us about how the brain works? Philosophical transactions of Royal Society biological sciences, 360, 1185-1205.

De Cruz, H. (2006). Why are some numerical concepts more successful than others? An evolutionary perspective on the history of number concepts. Evolution and Human Behavior, 27 (4), 306-323.

Dehaene, S., Bossini, S. \& Giraux, P. (1993). The mental representation of parity and numerical magnitude. Journal of Experimental Psychology: General, 122 (3), 371-396.

Dehaene, S. \& Cohen, L. (1997). Cerebral pathways for calculation:double dissociation between rote verbal and quantitative knowledge of arithmetic. Córtex, 33, 219-250.

Dehaene, S.; Dehaene-Lambertz, G. \& Cohen, L. (1998). Abstract representations of numbers in the animal and human brain. Trends in Neurosciences, 21 (8), 355-361.

Dehaene, S.; Molko, N.; Cohen, L \& Wilson, A.J. (2004). Arithmetic and the brain. Current Opinion in Neurobiology, 14, 218-224.

Dehaene S, Piazza M, Pinel P, Cohen L (2003) Three parietal circuits for number processing. Cognitive Neuopsychology, 20, 487-506.

De Smedt, B., Swillen, A., Devriendt, K., Fryns, J. P., Verschaffel, L., Boets, B. \& Ghesquière, P. (2007). Cognitive correlates of mathematical disabilities in children with Velo-Cardio-Facial Syndrome. Neuropsychologia, 45, 885-895.

Díaz, R. (2006). Innatismo y Cultura en el Aprendizaje de la Noción de Número. Revista Cognición, 5, 37-50. 
Dowker, A. (2006). What can functional brain imaging studies tell us about typical and atypical cognitive development in children?. Journal of physiology, 99, 333-341.

Ebersbach, M.; Luwel, K.; Frick, A.; Onghena, P. \& Verschaffel, L.(2008).The relationship between the shape of the mental numbernext term line and familiarity with previous termnumbersnext term in 5- to 9-year old children: Evidence fora segmented linear model. Journal of Experimental Child Psychology, 99 (1), 1-17.

Emmerton, J.; Lohmann, A. \& Niemann, J. (1997). Pigeons' serial ordering of numerosity with visual arrays. Animal Learning \& Behavior, 25, 234-244.

Feigenson, L., \& Carey, S. (2005). On the limits of infants' quantification of small object arrays. Cognition, 97, 295-313.

Feigenson, L.; Dehaene, S. \& Spelke, E. (2004). Core systems of number. Trends in Cognitive Sciences, 8, 307-314.

Fernández, J.A. (2006). Desarrollo del pensamiento logico-matemático. En: Educación Infantil: Orientaciones y recursos metodológicos para una enseñanza de calidad. J. Quintanal \& E. Miraflores (Coor.), 10, pp. 299-326.

Féron, J., Gentaz, E., \& Streri, A. (2006). Evidence of amodal representation of small numbers across visuo-tactile modalities in 5-month-old infants. Cognitive Development, 21, 81-92.

Fias, W.; Lammertyn, J.; Caessens, B. \& Orban G. (2007). Processing of abstract ordinal knowledge in the horizontal segment of the intraparietal sulcus. The Journal of Neuroscience, 27 (33), 8952-8956.

Flombaum, J; Junge, J. \& Hauser M. (2005). Rhesus monkeys spontaneously compute addition operations over large numbers. Cognition, 97 (3), 15-325.

Fodor, J. (1986). La modularidad de la mente. Madrid: Ediciones Morata.

Gallistel, R. \& Gelman, R. (1992). Preverbal and verbal counting and computation. Cognition, 44, 43-74.

Garcia, E. (2005). La construcción histórica de la psique. Sevilla: Eduforma.

Gelman, R. \& Gallistel, C. R. (1978). The child's understanding of number. Cambridge, MA: Harvard University Press. 
Gracia-Bafalluy, M. \& Noël, M. (2008). Does finger next term training increase young children's numerical performance?.Córtex, 44 (4), 368-375.

Gelman, R. \& Gallistel, C. (2004). Language and the origin of numerical concepts. Science, $306,441-443$.

Gerstmann, J. (1940). Syndrome of finger agnosia, disorientation for right and left, agraphia and acalculia. Archives of Neurology and Psychiatry, 44, 398-407.

Gevers, W. \& Lammertyn, J. (2005). The hunt for SNARC. Psychology Science, 47 (1), 1021.

Gilmore, C.K. \& Spelke, E. S. (en prensa). Children's understanding of the relationship between addition and subtraction. Cognition.

González-Hernández, A. (2007). Los números y otras secuencias se representan espacialmente. Ciencia Cognitiva: Revista Electrónica de Divulgación, 1:1, 10-11.

Göbel, S. \& Rushworth, M. (2004). Cognitive Neuroscience: Acting on Numbers. Current Biology (14), 517-519.

Gracia-Bafalluy, M. \& Noël, M. (2008). Does finger training increase young children's numerical performance?. Córtex, 44 (4), 368-375.

Halpern, C.; Glosser, G.; Clark, R.; Gee, J.; Moore, P.; Dennis, K.; McMillan, C.; Colcher, A. y Grossman, M. (2004). Dissociation of numbers and objects in corticobasal degeneration and semantic dementia. Neurology, 62, 1163-1169.

Hassenstein B., 1974. Otto Koehler--his life and his work. Zeitschrift fur Tierpsychologie, 35 (5), 449-464.

Hessl, D.; Rivera, S. y Reiss, A. (2004). The Neuroanatomy and Neuroendocrinology of Fragile X Syndrome. Mental Retardation and Developmental Disabilities Research Reviews, 10, 17-24.

Inhelder, B. \& Piaget, J. (1964). The early growth of logic in the child. Harper \& Row: New York.

Jordan, K. \& Brannon, E. (2006). Weber's Law influences numerical representations in rhesus macaques. (Macaca mulatta). Animal Cognition, 9, 159-172.

Kahn, H. (1991). Acalculia: an historical review of localization. Brain Cognition, 17, 102-15. 
Kamii, C. (1988). El niño reinventa la aritmética : implicaciones de la teoría de Piaget. Madrid: Visor.

Kamii, C.; Rummelsburg, J \& Kari, A. (2005). Teaching arithmetic to low-performing, lowSES first graders. The Journal of Mathematical Behavior, 24 (1), 39-50.

Kaufmann, L. (2008). Dyscalculia: neuroscience and education. Educational Research, 50 (2), $163-175$.

Kilian, A.; von Fersen L. \& Gunturkun, O. (2005). Left hemispheric advantage for numerical abilities in the bottlenose dolphin. Behavioural Processes, 68, (2), 179-184.

Klein, A.; Starkey, P. \& Ramirez, A.B. (2003). Pre-K Mathematics Curriculum. USA: Pearson Early Learning.

Klessinger, N.; Szczerbinskia, M. \& Varley, R. (2007). Algebra in a man with severe aphasia. Neuropsychologia, 45 (8), 1642-1648.

Knops, A.; Nuerk, H.; Sparing, R.; Foltys, H. \& Willmas, K. (2006). On the functional role of human parietal córtex in number processing: How gender mediates the impact of a 'virtual lesion' induced by rTMS, Neuropsychologia 44, 2270-2283.

Kobayashi, T., Hiraki, K., Mugitani, T. \& Hasegawa, T. (2004). Baby arithmetic: One object plus one tone. Cognition, 91 (2), 23-34.

Labinowicz, E. (1986). Introducción a Piaget. Pensamiento. Aprendizaje y Enseñanza. Mexico: Fondo Educativo Interamericano.

Le Bihanl, D. (2007). The 'wet mind': water and functional neuroimaging. Physics in medicine and biology, 52, 57-90.

Le Corre, M.; Van de Walle, G.; Brannon, E. M., \& Carey, S. (2006). Revisiting the competence/performance debate in the acquisition of the counting principles. Cognitive psychology, 52, 130-169.

Le Corre, M., \& Carey, S. (2007). One, two, three, four, nothing more: How numerals are mapped onto core knowledge of number in the construction of the counting principles. Cognition, 105 (2), 395-438.

Le Corre, M. \& Carey, S. (2008). Why the verbal counting principles are constructed out of representations of small sets of individuals: a reply to Gallistel. Cognition, 107 (2), $650-62$ 
Lebrun, Y. (2005). Gerstmann’s Syndrome. Journal of Neurolinguistics. 18, 317-326.

Lemer, C.; Dehaene, S.; Spelke, E. \& Cohen L (2003) Approximate quantities and exact number words: dissociable systems. Neuropsychologia, 41, 1942-1958.

Loetscher, T.; Bockisch, C.J. \& Brugger, P. (2008). Looking for the answer: The mind's eye in numbernext term space. Neuroscience, 151 (3), 725-729

Longo, M. \& Lourenco, S. (2007). Spatial attention and the mental number line: Evidence for characteristic biases and compression. Neuropsychologia, 45, 400-1407.

Lyon, B.(2003). Egg recognition and counting reduce costs of avian conspecific brood parasitism. Nature 422, 495-499

Mapelli, D.; Rusconi, E. \& Umilta, C. (2003). The SNARC effect: an instance of the Simon effect?. Cognition 88 (3), 1-10.

Molko, N.; Cachia, A.; Rivière, D.; Mangin, J.; Bruandet, M.; Le Bihan, D.; Cohen, L. \& Dehaene, S. (2003). Functional and atructural alterations of the intraparietal sulcus in a developmental dyscalculia of genetic origin. Neuron, 40 (4), 847-858.

Massardo T.; Jofre M.; Sierralta P.; Canessa J.; Gonzalez P.; Humeres P. y, Valdebenito R. (2007). Chilean experience with the use of F-18-deoxyglucose positron emission tomography (Valdebenito, Robert). Revista médica de Chile, 135, (3), 375-383.

Moyer, R. S., \& Landauer, T. K. (1967). Time required for judgements of numerical inequality. Nature, 215, 1519-1520.

Noël, M.P. (2005). Finger gnosia: a predictor of numerical abilities in children?. Child Neuropsychology, 11, 413-430.

Pesenti, M.; Thioux, M.; Seron, X. \& De Volder, A. (2000). Neuroanatomical substrates of Arabic number processing, numerical comparison, and simple addition: A PET Study. Journal of Cognitive Neuroscience, 12,(3), 461-479.

Piaget, J. (1980). Biología y conocimiento. Madrid: Siglo Veintiuno.

Piaget, J. \& Szeminska, A. (1965). The child's conception of number. Routledge and Kegan Paul: London.

Pinel P.; Piazza, M.; Le Bihan, D. \& Dehaene, S. (2004). Distributed and overlapping cerebral representations of number, size, and luminance during comparative judgments. Neuron 41, 983-993. 
Piazza M.; Pinel, P.; Le Bihan, D. \& Dehaene, S. (2007). A magnitude code common to numerosities and number symbols in human intraparietal córtex. Neuron 53 (2), 293-305

Polk T, Reed C, Keenan J, Hogard P, Anderson CA (2001) A dissociation between symbolic number knowledge and analogue magnitude information. Brain and Cognition 47 (3), $545-563$.

Raichle, M. (2003). Functional Brain Imaging and Human Brain Function. The journal of neuroscience, 23 (10), $3959-3962$.

Restle, F. (1970). Speed of adding and comparing numbers. Journal of Experimental Psychology, 95, 437-444.

Rubinsten, O.; Henik, A.; Berger, A. \& Shahar-Shalev, S. (2002). The development of internal representations of magnitude and their association with arabic numerals. Journal of Experimental Child Psychology 81, 74-92.

Ruesga, M.P. (2004). Las matemáticas a través del juego. Burgos: Mª P. Ruesga (Editor).

Rugani, R.; Regolin, L. \& Vallortigara, G. (2007).Rudimental numerical competence in 5day-old domestic chicks (Gallus gallus): Identification of ordinal position. Journal of Experimental Psychology: Animal Behavior Processes 33, (1), 21-31.

Rusconi, E.; Kwana, B.; Giordano, B.L.; Umilta, C. \& Butterworth, B. (2006). Spatial representation of pitch height: the SMARC effect. Cognition, 99, 113-129.

Rusconi, E.; Walsh, V. \& Butterworth, B. (2005). Dexterity with numbers: rTMS over left angular gyrus disrupts finger gnosis and number processing. Neuropsychologia $43,1609-1624$.

Saal, A.; Izquierdo, E. \& Codnia, J. (2005). Efecto SNARC con estímulos simbólicos sensoriales, Anales AFA, 17, 337-341.

Sarnecka, B. W.; Kamenskaya, V. G.; Yamana, Y.; Ogura, T. \& Yudovina, Y.B. (2006).From grammatical number to exact numbers: Early meanings of 'one', 'two', and 'three' in English, Russian, and Japanese. Cognitive psychology, 55 (2), 136-68.

Silvanto,J.; Muggleton, N.; Cowey, A. \& Walsh, V. (2007). Neural activation state determines behavioral susceptibility to modified theta burst transcranial magnetic stimulation. European journal of neuroscience, 26, 523-528. 
Shalev, R.S. (2004). Developmental dyscalculia. Journal of child neurology, 19 (10), 765 771.

Shelli, R.; Kesler, S.; Menon, V. \& Reiss, A. (2006). Neurofunctional Differences Associated with Arithmetic Processing in Turner Syndrome. Cerebral Córtex, 16, 849-856.

Sheridan, S.R. (2005). A theory of marks and mind: the effect of notational systems on hominid brain evolution and child development with an emphasis on exchanges between mothers and children. Medical Hypotheses, 64 (2), 417-427.

Spelke, E.S. \& Kinzler, K. D. (2007). Core knowledge. Developmental Science, 10 (1), 89-96.

Stippich, C.; Rapps, N.; Dreyhaupt, J.; Durst, A.; Kress, B.; Nennig, E.; Tronnier, V. \& Sartor, K. (2007). Localizing and lateralizing language in patients with brain tumors: feasibility of routine preoperative functional MR imaging in 81 consecutive patients. $R a-$ diology, 243, 828-836.

Thiouxa, M.; Pesentia, M.; Costesb, N.; De Volderc, A. \& Serona, X. (2005). Taskindependent semantic activation for numbers and animals. Cognitive Brain Research $24,284-290$.

Trianes, M. \& Gallardo, J. (2004). Definición, campos y proceso histórico de la psicología de la educación y del desarrollo. En Psicología de la educación y del desarrollo en contextos escolares. Madrid: Piramide.

VanMarle, K.; Aw, J.; McCrink, K. \& Santos, L. (2006). How capuchin monkeys (Cebus apella) quantify objects and substances. Journal of Comparative Psychology 120 (4), 416-426.

von Aster, M. G. \& Shalev, R. (2007). Number development and developmental dyscalculia. Developmental Medicine \& Child Neurology, 49 (11), 868-873.

Ward, C. \& Smuts B. (2007). Quantity-based judgments in the domestic dog (Canis lupus familiaris). Animal Cognition 10 (1), 71-80.

Weber (2007). Weber's law. In Encyclopædia Britannica. Retrieved September 6, 2007, from Encyclopædia Britannica Online: http://www.britannica.com/eb/article-9076393.

William, J (1890). Principles of Psychology. New York: Henry Holt \& Company, Vol. 1, 28.

Willburger, E.; Fussenegger, B.; Moll, K.; Wood, G. \& Landerl, K. (2008). Naming speed in dyslexia and dyscalculia. Learning and Individual Differences, 18 (2), 224-236. 
Wilson, A. J.; Revkin, S. K.; Cohen, R.; Cohen, L. \& Dehaene, S. (2006). An open trial assessment of "The Number Race", an adaptive computer game for remediation of dyscalculia. Behavioral and Brain Functions, 30, 2-20.

Wynn, K. (1992a). Addition and subtraction by human infants. Nature, 358, 749-750.

Wynn, K. (1992b). Children's acquisition of number words and the counting system. Cognitive Psychology, 24, 220-251.

Woods, A.; Mennemeier, M.; Garcia-Rill, E.; Meythaler, J.; Mark, V.; Jewel. G. \& Murphy, H. (2006) Bias in magnitude estimation following left hemisphere injury. Neuropsychologia, 44,1406-1412.

Xu, F.; Spelke, E.S. \& Goddard, S. (2005). Number sense in human infants. Developmental Science, 8 (1), 88-101.

Xu, F. \& Arriaga, R. (2007). Number discrimination in 10-month-old infants. British journal of developmental psychology, 25, 103-108.

Zebian, S. (2005). The SNARC Effect and the REVERSE SNARC Effect in English and Arabic Monoliterates, Biliterates, and Illiterate Arabic Speakers. Journal of Cognition and Culture, 5, 165-190. 